\title{
Optical focusing of Bose-Einstein condensates
}

\author{
R. Richberg, ${ }^{1,2,{ }^{*}}$ S. S. Szigeti, ${ }^{1}$ and A. M. Martin $\odot^{2}$ \\ ${ }^{1}$ Department of Quantum Science, Research School of Physics, The Australian National University, Canberra, ACT 2601, Australia \\ ${ }^{2}$ School of Physics, University of Melbourne, Melbourne 3010, Australia
}

(Received 8 December 2020; revised 11 May 2021; accepted 21 May 2021; published 14 June 2021)

\begin{abstract}
We theoretically investigate the optical focusing of a rubidium Bose-Einstein condensate onto a planar surface. Our analysis uses a Gaussian variational method that includes the effects of two-body atom-atom interactions and three-body recombination losses. The essential factors such as the width, peak density, and atom loss rate of the focused BEC profile on the surface are investigated and compared to Gross-Pitaevskii numerical simulations. We find a reasonable agreement in the results between our analytical approach and the numerical simulations. Our analysis predicts that condensates of $10^{5}$ atoms could be focused down to $\sim 10 \mathrm{~nm}$ widths, potentially allowing nanometer-scale atomic deposition with peak densities greater than $10^{5}$ atoms $/ \mu \mathrm{m}^{2}$.
\end{abstract}

DOI: 10.1103/PhysRevA.103.063304

\section{INTRODUCTION}

Atom lithography aims to deposit nanostructures onto a surface via the direct manipulation of cold-atom beams with optical fields $[1,2]$. It potentially offers a controlled and flexible deposition procedure at the atomic scale, which could increase the density of transistors in computer chips $[3,4]$. Early theoretical works that detailed how optical beams could be used to focus atomic beams to nanoscale spot sizes [5-7] were soon followed by experimental demonstrations of direct depositions with sodium [1] and chromium [2] atoms in which a $60 \mathrm{~nm}$ resolution was achieved. Direct atomic deposition has also been demonstrated with ytterbium [8] and iron $[9,10]$ (which, respectively, resulted in 93 and $95 \mathrm{~nm}$ profile resolutions) and is capable of the precise generation of two-dimensional (2D) and 3D nanostructures [11].

Almost all of the experiments accomplished so far have used an oven source of atoms in which the beam is collimated with an aperture followed by a transverse laser cooling process [2] before traveling through a focusing potential. However, there are advantages to using a Bose-Einstein condensate (BEC) of neutral atoms for atom deposition. Since the de Broglie wavelength of an atomic gas is of the order of the mean distance between particles, a BEC source would bring atoms to wavelengths between $1 \mathrm{~nm}$ and $1 \mathrm{pm}$ for nano-Kelvin temperatures [12], resulting in an excellent collimation of the beam of atoms as well as a high flux density [13]. Using a BEC source can also reduce effects such as chromatic aberration and angular divergence [14], with the longitudinal and transverse velocity distributions typically being much narrower when incident on the surface compared to those resulting from oven or thermal sources.

There have been efforts to study the manipulation of BECs using different techniques. In 1996, a work on matter wave focusing was theoretically conducted [15] where, using the

*Formerly known as Amir M. Kordbacheh. trapped BEC, they tried to scale the focusing times for the ideal and interacting $\mathrm{BEC}$ to prove that an interacting $\mathrm{BEC}$ can focus more quickly. In another effort, the diffraction of a BEC from a solid grating was probed by analyzing the Casimir-Polder interaction of atoms with a solid grating in the presence of an external laser source [16]. In 2011, the interaction of Bose-Einstein condensates with the optical near field above plasmonic micro- and submicrometer structures was investigated; the strength of optical near fields was measured by observing the reflection of cold atoms from the surface [17]. In [18], the coherent state-selective patterning of matter waves beyond the diffraction limit was analyzed using subwavelength localization via an adiabatic passage technique. Then, further discussion regarding the implementation of this approach in nanolithography using $\mathrm{Ne}$ and ${ }^{87} \mathrm{Rb}$ was carried out. Later, in a similar work [19], utilizing the same technique as above, a single-site addressing implementation of ultracold neutral atoms was considered by loading them into a two-dimensional optical lattice; using the subwavelength localization via adiabatic passage offers a higher addressing resolution and other benefits such as robustness against parameter variations and coherence of the transfer process. Last but not least, research was performed in 2010 regarding matter wave focusing of BECs using alkali atoms [20]. Theoretically, they used a different approach than using optical light fields to focus neutral atoms. Using Frensel zone plates (FZPs), they studied the compression of a BEC when passing through the etched holes, and they showed that the focused deposition of alkali atoms by ZPs can create quantum electronic components on the $50 \mathrm{~nm}$ scale, comparable to that attainable by ion beam implantation. Providing simple, quantitative estimates of the deposited focused widths and peak densities of a BEC source using a light field is the subject of this paper.

For thermal atomic sources, the atomic density is sufficiently dilute that interatomic interactions are negligible. Consequently, a classical approach based on single-atom trajectories is suitable in situations where the wavelike properties 
of the atoms are not too important, in analogy to ray optics [21], with some wavelike effects such as diffraction and chromatic aberration accounted for in an ad hoc manner [22-24]. However, the effect of interactions must be accounted for in BEC sources. Interatomic interactions in a BEC are dominated by $s$-wave scattering [25-28] and, in certain atomic species, can be tuned from strongly repulsive to strong attractive via a Feshbach resonance [29]. The matter wave focusing dynamics of trapped BECs in both repulsively interacting and noninteracting regimes have been previously investigated [30]. The significance of our work is to take the atomic interaction into account when focusing a freely propagating $\mathrm{BEC}$ and to scale its effect on the broadening of the nano-focal-spot sizes and peak densities achievable in realistic nanolithography experiments with cold atoms. The other substantial point considered in this work is the effect of three-body recombination losses [31-33] on the focusing scheme. This effect becomes especially striking in the high flux regimes of focus. The influence of three-body losses can be controlled via the sign and strength of the $s$-wave interactions.

In this paper, we use an analytical variational approach based on a Gaussian ansatz [34] to model the focusing dynamics of a freely falling BEC. Our model assumes a harmonic optical focusing potential, with optimal focal parameters determined via a classical particle trajectories approach, [14] and includes two-body atom-atom interactions and three-body recombination losses. We obtain estimates of the deposited focused BEC widths and peak densities leading to a resolution of about $10 \mathrm{~nm}$ via the variational method. We also present the corresponding Gross-Pitaevskii equation (GPE) numerical results to test the validity of the Gaussian ansatz approach.

\section{PROBLEM DESCRIPTION}

The problem we consider is illustrated schematically in Fig. 1. A cloud of ${ }^{85} \mathrm{Rb}$ atoms is initially confined by a harmonic trap potential at $t=0$. Having this potential turned off abruptly, the released BEC starts expanding while propagating freely along the vertical $z$ axis, while it is approaching the tight harmonic focusing potential along the $x$ axis. It then travels through the potential and is focused down to a nanometer structure along the $x$ axis, where it is deposited on a surface.

We begin by defining the time-dependent nonlinear Schrödinger equation (i.e., the GPE) [35-38] in 3D,

$$
\begin{aligned}
i \hbar \frac{\partial \psi(\mathbf{r}, t)}{\partial t}= & {\left[-\frac{\hbar^{2}}{2 m} \nabla_{r}^{2}+V_{\mathrm{ext}}(\mathbf{r}, t)+u \psi|(\mathbf{r}, t)|^{2}\right.} \\
& \left.-i K \psi|(\mathbf{r}, t)|^{4}\right] \psi(\mathbf{r}, t),
\end{aligned}
$$

where $\hbar$ and $m$ are Planck's constant and the atomic mass for rubidium 85, respectively. The first nonlinear term, $u|\psi(\mathbf{r}, t)|^{2}$, is the mean-field potential term, where $u=\frac{4 \pi \hbar^{2} a_{s}}{m}$ is the interatomic interaction strength, $|\psi(\mathbf{r}, t)|^{2}$ is the atomic density, and $a_{s}$ is the $s$-wave scattering length $[25,27,39,40]$. The value $a_{s}$ can be tuned, in practice, from positive (repulsive interactions) to negative (attractive interactions) values through the use of a Feshbach resonance [29,41,42]. The second nonlinear term governs three-body recombination losses.

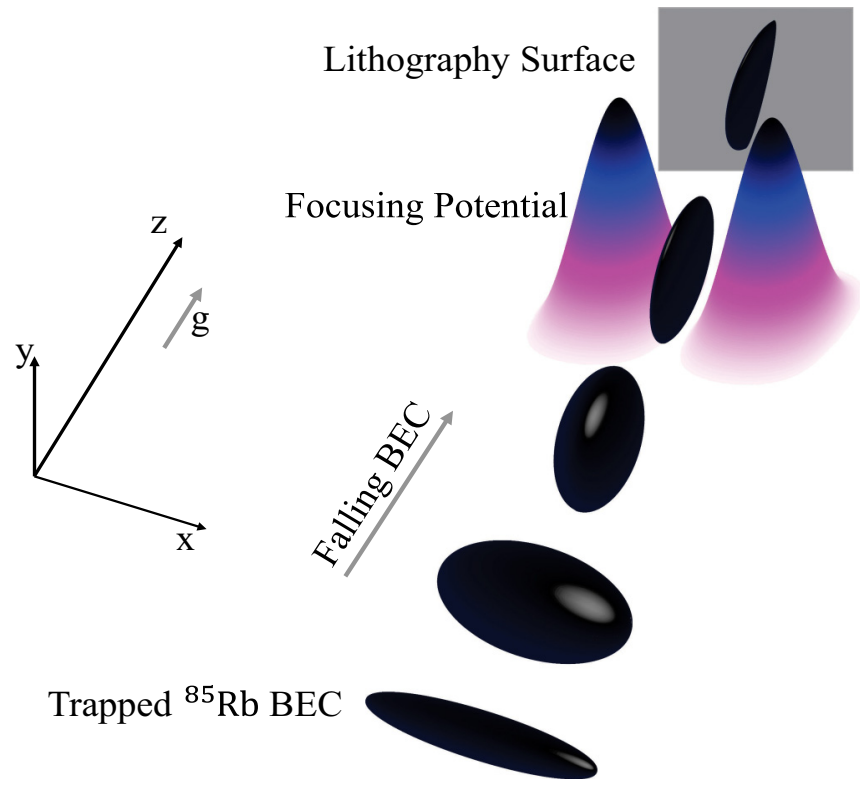

FIG. 1. 3D schematic illustration of atom deposition using a ${ }^{85} \mathrm{Rb}$ BEC focused by an optical potential, assumed to be approximately harmonic. The BEC falls along the $z$ axis, which is the direction of gravitational acceleration.

In this work, we set the three-body loss rate coefficient to $K=4 \times 10^{-41} \mathrm{~m}^{6} \mathrm{~s}^{-1}$, consistent with experimentally determined values for ${ }^{85} \mathrm{Rb}$ condensates [43-45]. At $t=0$, the condensate is initially confined and held by a harmonic trap, $V_{\text {ext }}(\mathbf{r}, t=0)=m\left[\omega_{0 x}^{2} x^{2}+\omega_{0 y}^{2} y^{2}+\omega_{0 z}^{2}\left(z-z_{0}\right)^{2}\right] / 2$, with $z_{0}$ being the initial distance between the center of mass of the condensate and the center of the focusing potential, and $\omega_{0 x}$, $\omega_{0 y}$, and $\omega_{0 z}$ are the initial trapping frequencies along the $x$, $y$, and $z$ axes, respectively, at $t=0$. For $t>0$, the confining potential is switched off and the focusing parabolic potential is switched on. We assume an optical focusing potential, which induces a dipole moment in the atoms of the BEC. The interaction between the dipole moment and the electric field causes a dipole force [46] with a gradient towards the nodes or antinodes of the laser intensity. The focusing potential generated by a laser of intensity $I(x, z)$ is [47]

$$
U_{\mathrm{dip}}(x, z)=\frac{\hbar \Delta}{2} \ln \left[1+\frac{\gamma^{2}}{\gamma^{2}+4 \Delta^{2}} \frac{I(x, z)}{I_{s}}\right],
$$

where $\Delta$ denotes the detuning of the laser frequency from the atomic resonance, $\gamma=38 \mathrm{MHz}$ the natural linewidth of the $D_{2}$ atomic transition of ${ }^{85} \mathrm{Rb}$ (i.e., spontaneous decay rate), and $I_{s}=1.67 \mathrm{~mW} / \mathrm{cm}^{2}$ is the saturation intensity of this transition. An approximately harmonic potential along $x$ can be engineered using a spatial light modulator $[48,49]$. Assuming a Gaussian beam profile along $z$ (see Fig. 1), this gives $I(x, z)=I_{0} \exp \left(-2 z^{2} / \sigma_{z}^{2}\right)\left(k^{2} x^{2}\right)$, where $I_{0}$ is the maximum intensity of the spatially varying harmonic profile, $\sigma_{z}$ is the radius of the beam at $1 / e^{2}$ value of the maximum intensity, and $k$ determines the strength of the harmonic focusing.

To study the evolving BEC in a focusing potential, we assume that the BEC is located in a stationary frame at $z=0$, while the harmonic potential is in a moving frame 
approaching the BEC. In this frame, Eq. (2) is

$$
U_{\text {dip }}(x, t)=\frac{\hbar \Delta}{2} \ln \left[1+\frac{\gamma^{2}}{\gamma^{2}+4 \Delta^{2}} \frac{I_{0}}{I_{s}} k^{2} x^{2} f(t)\right],
$$

where

$$
f(t)=\exp \left\{\frac{-2}{\sigma_{z}^{2}}\left[z_{0}-z(t)\right]^{2}\right\},
$$

where $z(t)=\frac{1}{2} g t^{2}+v_{0} t$ is the varying distance in terms of time, which depends on the gravitational acceleration $g$ and initial velocity $v_{0}$ imparted to the BEC. For the relatively low values of intensity, $I_{0}$, and relatively large values of the detuning, $\Delta$, Eq. (3) reduces to

$$
U_{\mathrm{dip}}(x, t) \approx \frac{\hbar \Delta \gamma^{2}}{\left(\gamma^{2}+4 \Delta^{2}\right)} \frac{I_{0}}{I_{s}} k^{2} x^{2} f(t) .
$$

In this regime, Eq. (5) takes the form of a harmonic potential such that $V_{\mathrm{ext}}(x, t>0)=\frac{1}{2} m \omega^{2}(t) x^{2}=U_{\mathrm{dip}}(x, t)$, with timedependent frequency,

$\omega_{x}^{2}(t)=\frac{\hbar \Delta \gamma^{2} k^{2}}{m\left(\gamma^{2}+4 \Delta^{2}\right)} \frac{I_{0}}{I_{s}} \exp \left\{\frac{-2}{\sigma_{z}^{2}}\left[z_{0}-\left(\frac{1}{2} g t^{2}+v_{0} t\right)\right]^{2}\right\}$.

\section{THE VARIATIONAL APPROACH}

In this section, we consider a variational approach based on the GPE to model the BEC dynamics. We adapt the approach considered in Refs. [34,50] to account for the effect of threebody losses to the BEC dynamics in focusing regimes.

To begin, we note that the GPE wave function that describes our BEC minimizes the action [51],

$$
S=\int \mathcal{L}_{\text {tot }}(\mathbf{r}, t) d^{3} \mathbf{r} d t
$$

where $\mathcal{L}_{\text {tot }}$ is the total Lagrangian density [52],

$$
\begin{aligned}
\mathcal{L}_{\text {tot }}(\mathbf{r}, t)=\mathcal{L}+\mathcal{L}_{R}= & \frac{i \hbar}{2}\left(\psi * \frac{\partial \psi}{\partial t}-\psi \frac{\partial \psi^{*}}{\partial t}\right)-\frac{\hbar^{2}}{2 m}|\nabla \psi|^{2} \\
& -V_{\text {ext }}(\mathbf{r}, t)|\psi|^{2}-\frac{g}{2}|\psi|^{4}+\mathcal{L}_{R},
\end{aligned}
$$

where $\mathcal{L}_{R}$ is the Lagrangian density for the three-body recombination term in Eq. (1), defined by

$$
\mathcal{L}_{R}=-\frac{1}{3}\left(R \psi^{*}\right)
$$

where

$$
R\left(\psi, \psi^{*}\right)=-i K|\psi|^{4} \psi .
$$

We note that the loss rate due to the two-body spin relaxation (two-body losses) between atoms in confined BECs is negligible in relatively high-density regimes compared to the three-body recombination losses [53-56]; hence, the contribution of two-body loss is not considered in the GPE [Eq. (1)] and in the total Lagrangian density [Eq. (8)]. In order to minimize the GPE action in Eq. (7), we choose an appropriate single-particle trial wave function. A good choice is the following Gaussian variational ansatz:

$$
\begin{aligned}
\psi_{0}(x, y, z, t) & \\
= & \mathcal{A}(t) \exp \left(\sum_{r=x, y, z}\right. \\
& \left.\times\left\{-\frac{\left[r-r_{0}(t)\right]^{2}}{2 \mathcal{W}_{r}^{2}(t)}+i\left[\alpha_{r}(t) r+\beta_{r}(t) r^{2}+\phi_{r}(t)\right]\right\}\right),
\end{aligned}
$$

with the normalization factor

$$
A(t)=\frac{\sqrt{N(t)}}{\sqrt{\pi^{\frac{3}{2}} \mathcal{W}_{x}(t) \mathcal{W}_{y}(t) \mathcal{W}_{z}(t)}},
$$

where $\mathcal{W}_{r}, r_{0}, \beta_{r}, \alpha_{r}$, and $\phi_{r}$ are the variational parameters. $\mathcal{W}_{r}$ corresponds to the respective condensate width, $r_{0}$ is the initial position of the BEC center, $\beta_{r}$ indicates the (curvature radius $)^{-1 / 2}, \alpha_{r}$ represents the slope, and $\phi_{r}$ describes the phase of the condensate. The term $N(t)$ represents the number of atoms in the BEC, which changes with time. This follows from $\int d^{3} \mathbf{r}\left|\psi_{0}(\mathbf{r}, t)\right|^{2}$, which is not necessarily conserved due to three-body losses. We set the center of the condensate to the center of the Cartesian coordinates, $\left(x_{0}, y_{0}, z_{0}\right)=(0,0,0)$.

The objective is to find the equations of motion for these parameters. Substituting Eq. (8) and Eq. (11) into the average Lagrangian density, $L_{\text {tot }}(t)=\int d^{3} \mathbf{r} \mathcal{L}_{\text {tot }}(\mathbf{r}, t)$, we obtain (see Appendix 1 for details)

$$
\begin{aligned}
& L_{\mathrm{tot}}(t) \\
& =-\sum_{r=x, y, z} \frac{|A|^{2} \mathcal{W}_{r} \sqrt{\pi}}{2}\left[\hbar \dot{\beta}_{r} \mathcal{W}_{r}^{2}+2 \hbar \dot{\phi}_{r}+\frac{\hbar^{2}}{2 m}\left(\frac{1}{\mathcal{W}_{r}^{2}}\right)\right. \\
& \left.\quad+\frac{\hbar^{2}}{2 m}\left(4 \beta_{r}^{2} \mathcal{W}_{r}^{2}\right)+\frac{\hbar^{2}}{2 m}\left(2 \alpha_{r}^{2}\right)+\frac{1}{2} m \omega_{x}^{2}(t) \mathcal{W}_{x}^{2}+\frac{g|A|^{2}}{\sqrt{2}}\right] \\
& \quad+L_{R}(t),
\end{aligned}
$$

where $L_{R}(t)=\int d^{3} \mathbf{r} \mathcal{L}_{R}(\mathbf{r}, t)$. The equations of motion for all variational factors are given by the Euler-Lagrange equations for real and imaginary components of $L$. Since the imaginary part is trivial, one can ignore it and only consider the contribution due to the real components,

$\frac{d}{d t} \frac{\partial L}{\partial \dot{q}_{i}}-\left[\frac{\partial L}{\partial q_{i}}+\operatorname{Re}\left(\frac{\partial L_{R}}{\partial q_{i}}\right)\right]=0 ; q \in\left\{\mathcal{A}(t), \mathcal{W}_{r}, \alpha_{r}, \beta_{r}, \phi_{r}\right\}$,

where $\frac{\partial L_{\text {tot }}}{\partial \dot{q}_{i}}=\frac{\partial L}{\partial \dot{q}_{i}}$ since $\frac{\partial L_{R}}{\partial \dot{q}_{i}}=0$. Since $\frac{\delta \mathcal{L}_{R}}{\delta \psi^{*}}=-\frac{1}{3} R$, we can write

$$
\operatorname{Re}\left(\frac{\partial \mathcal{L}_{R}}{\partial q_{i}}\right)=-\frac{1}{6}\left(R \frac{\partial \psi^{*}}{\partial q_{i}}+R^{*} \frac{\partial \psi}{\partial q_{i}}\right) .
$$

Integrating both sides of Eq. (15) and inserting the associated result into Eq. (14) gives

$$
\frac{d}{d t} \frac{\partial L}{\partial \dot{q}_{i}}-\frac{\partial L}{\partial q_{i}}=-\frac{1}{6} \int\left(R \frac{\partial \psi^{*}}{\partial q_{i}}+R^{*} \frac{\partial \psi}{\partial q_{i}}\right) d^{3} \mathbf{r} .
$$


Solving Eq. (16) for $q_{i}=\mathcal{A}, \mathcal{W}_{r}, \alpha_{r}, \beta_{r}, \phi_{r}$ results in the following dimensionless variational equations for the BEC width dynamics and loss rate (see Appendix 2 for details):

$$
\begin{gathered}
\frac{d^{2} \mathcal{W}_{x}}{d t^{2}}+\omega_{x}^{2}(t) \mathcal{W}_{x}=\frac{\hbar^{2}}{m^{2}}\left(\frac{1}{\mathcal{W}_{x}^{3}}\right)+\frac{g N}{m(2 \pi)^{3 / 2} \mathcal{W}_{x}^{2} \mathcal{W}_{y} \mathcal{W}_{z}} \\
\quad-\frac{7 K^{2} N^{4}}{3(3 \pi)^{6} \hbar^{2} \mathcal{W}_{x}^{3} \mathcal{W}_{y}^{4} \mathcal{W}_{z}^{4}} \\
\frac{d^{2} \mathcal{W}_{y}}{d t^{2}}=\frac{\hbar^{2}}{m^{2}}\left(\frac{1}{\mathcal{W}_{y}^{3}}\right)+\frac{g N}{m(2 \pi)^{3 / 2} \mathcal{W}_{x} \mathcal{W}_{y}^{2} \mathcal{W}_{z}} \\
\frac{d^{2} \mathcal{W}_{z}}{d t^{2}}=\frac{\hbar^{2}}{m^{2}}\left(\frac{1}{\mathcal{W}_{z}^{3}}\right)+\frac{7 K^{2} N^{4}}{m(2 \pi)^{3 / 2} \hbar^{2} \mathcal{W}_{x}^{4} \mathcal{W}_{y}^{3} \mathcal{W}_{z}^{4}} \\
-\frac{7 K^{2} N_{z}^{2}}{3(3 \pi)^{6} \hbar^{2} \mathcal{W}_{x}^{4} \mathcal{W}_{y}^{4} \mathcal{W}_{z}^{3}} \\
\frac{d N(t)}{d t}=-\frac{K N^{3}}{9 \sqrt{3} \pi^{3} \hbar \mathcal{W}_{x}^{2} \mathcal{W}_{y}^{2} \mathcal{W}_{z}^{2}}
\end{gathered}
$$

with the initial conditions

$$
\begin{gathered}
\mathcal{W}_{x}(t=0)=\mathcal{W}_{0 x} ; \mathcal{W}_{y}(t=0)=\mathcal{W}_{0 y} ; \mathcal{W}_{z}(t=0)=\mathcal{W}_{0 z} ; \\
\dot{\mathcal{W}}_{x}(t=0)=\dot{\mathcal{W}}_{0 x} ; \dot{\mathcal{W}}_{y}(t=0)=\dot{\mathcal{W}}_{0 y} ; \dot{\mathcal{W}}_{z}(t=0)=\dot{\mathcal{W}}_{0 z} \\
N(t=0)=N_{0}
\end{gathered}
$$

We estimate the initial BEC widths along all three Cartesian axes, $\mathcal{W}_{0 x}, \mathcal{W}_{0 y}, \mathcal{W}_{0 z}$, using [27]

$$
\mathcal{W}_{0 i}=\left(\frac{2}{\pi}\right)^{1 / 10}\left(\frac{N_{0} a_{s}}{l}\right)^{1 / 5} \frac{\omega_{0}}{\omega_{i}} l \quad(i=x, y, z),
$$

in which we have introduced the harmonic oscillator length $l=\sqrt{\frac{\hbar}{m \omega_{0}}}$, where $\omega_{0}=\left(\omega_{0 x} \omega_{0 y} \omega_{0 z}\right)^{1 / 3}$. This estimate is obtained by minimizing the energy of a 3D Gaussian ansatz and neglecting the kinetic energy contribution, which is small for strong repulsive initial interactions.

\section{OPTIMAL POWER FOR FOCUSING}

By treating the atom dynamics as classical particle trajectories and neglecting the $y$ axis due to the transitional symmetry of the problem, the classical equations of motion for atomic trajectories along the $x$ and $z$ axes are given by [14]

$$
\begin{aligned}
& \frac{d^{2} x}{d t^{2}}+\frac{1}{m} \frac{\partial U(x, z)}{\partial x}=0, \\
& \frac{d^{2} z}{d t^{2}}+\frac{1}{m} \frac{\partial U(x, z)}{\partial z}=0 .
\end{aligned}
$$

To achieve the optimal power for focusing, first we combine Eqs. (23) and (24) using conservation of energy. Second, we consider the paraxial approximation [14] in which the wavelength of the focusing potential is considered to be much larger than the dimension of the falling atoms (i.e., the BEC width) along the $x$ axis, $k x \ll 1$. The approximation neglects aberrations (i.e., the trajectories are assumed to be perfectly parallel to the $z$ axis when falling towards the lattice). That said, one obtains the following second-order differential equation:

$$
\frac{d^{2} x}{d z^{2}}+q^{2} \exp \left(-2 z^{2} / \sigma_{z}^{2}\right) x=0
$$

where $q^{2}=\frac{\hbar \Delta}{2 E_{0}} \frac{I_{0}}{I_{s}} \frac{\gamma^{2}}{\gamma^{2}+\Delta^{2}} k^{2}$ and $E_{0}$ represents the kinetic energy of the atoms when they hit the focus (i.e., the lithographic surface). Since the power of a Gaussian laser beam relates to the peak intensity via $I_{0}=8 P_{0} / \pi \sigma_{z}^{2}$, the laser power needed to focus the atoms at any desired spot along the focal axis ( $z$ axis) can be determined,

$$
P_{0}=\xi \frac{\pi}{4} \frac{E_{0}}{\hbar \Delta} \frac{\gamma^{2}+4 \Delta^{2}}{\gamma^{2}} \frac{I_{s}}{k^{2}},
$$

where $\xi=q^{2} \sigma_{z}^{2}$ is a dimensionless parameter. A value of $\xi=$ 5.37 , determined by solving the classical equations of motion for atomic trajectories, optimally focuses the atoms onto the plane $z=0$ and $x=0$ (the center of the focusing potential). The selection of lower values of $\xi$ (smaller powers) leads to focusing on planes $z<0$.

Equation (26) indicates that the optimal power is a function of atomic kinetic energy. Since the atoms (in our case, the BEC) are accelerated over the traveling path due to gravity, the optimal power becomes dependent on the traveling distance, $z_{0}$. Hence, the larger $z_{0}$, the higher velocity of the BEC at the focus point, which consequently necessitates a relatively greater value of power for optimal focusing.

\section{ESTIMATE OF DEPOSITED 2D DENSITY DISTRIBUTION}

Suppose we have a substrate placed at the $z=0$ plane. We consider an ideal atom lithographic scenario, where atoms that intersect the $z=0$ plane are deemed deposited on the substrate surface. While in this case the lithographic surface might interfere with the focusing light field and cause disruption (i.e., diffraction and reflection at the surface), practical solutions are suggested in [2] rectifying this barrier. A time integral of the density profile at $z=0$ therefore provides a simple estimate of the total 2D distribution of atoms deposited on this surface,

$$
n_{0}(x, y)=\int_{0}^{t_{\mathrm{end}}}|\psi(x, y, t, z=0)|^{2} d t,
$$

where $t_{\text {end }}$ is the duration of the atom lithographic process. This estimate neglects the effect of the surface itself on the BEC dynamics. For example, as atoms are deposited on the surface, they leave the condensate and lower the overall meanfield energy. Furthermore, atoms that are not deposited will be reflected, potentially impacting the focusing dynamics. Nevertheless, the estimate of the deposited 2D density distribution provided by Eq. (27) can be considered a "best case."

Our goal is to determine atomic and focusing potential parameters that result in narrow deposited distributions with a high peak density. These can be estimated from Eq. (26) via the full width at half maximum (FWHM) of $n_{0}(x, y)$ and $\max _{x, y} n_{0}(x, y)$, respectively. Throughout this paper, we 
(a)

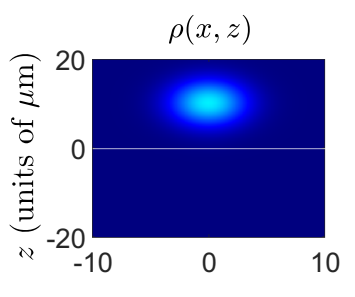

$n_{0}(x, y)$

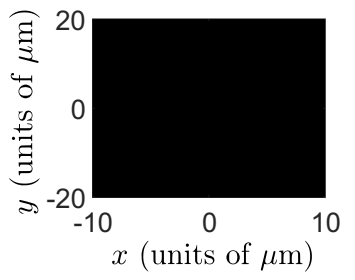

(f) (b)

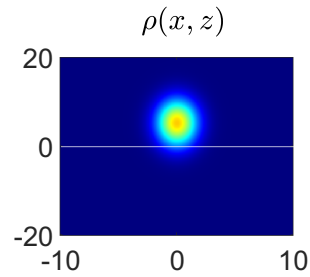

$n_{0}(x, y)$

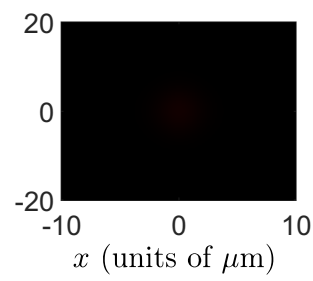

(g) (c)

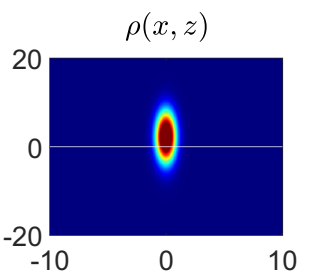

$n_{0}(x, y)$

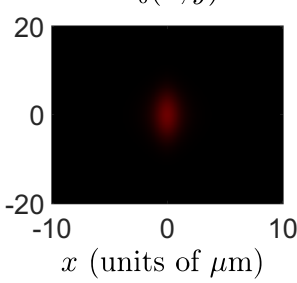

(h) (d)

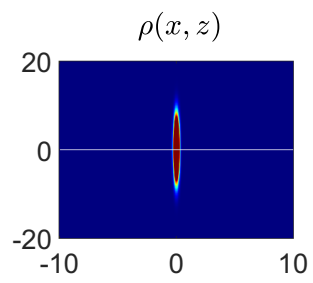

$n_{0}(x, y)$

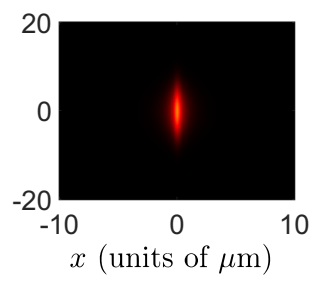

(i) (e)
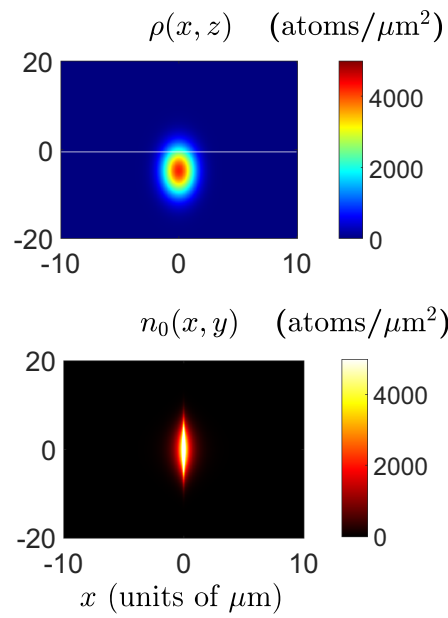

(j)

FIG. 2. Upper row: The cross-section view of the evolving BEC column density $\rho(x, z)=\int d y \rho(x, y, z)$ in the plane of ( $\left.x, z\right)$. (a)-(d) The procedure of focusing when the center of the BEC is located at $z=10,5,2,0 \mu \mathrm{m}$, respectively (from left to right). (e) The expansion of the BEC at $z=-5 \mu \mathrm{m}$ after it has left the focal spot (i.e., focal surface, $z=z_{f}=0$ ). Lower row: The corresponding integrated density distributions $n_{0}(x, y)$ at the focal surface $z=0$ (where the substrate is essentially placed). The value of $n_{0}(x, y)$ reaches its maximum when all atoms of the cloud have been deposited on the focal surface. All the simulations have been conducted using the variational analysis. The momentum kick in the simulations is set to zero and $a_{s}=-1 a_{0}$. Parameters are $N_{0}=10^{5}, \omega_{0 x}=2 \pi \times 10 \mathrm{~Hz}, \omega_{0 y}=\omega_{0 z}=2 \pi \times 70 \mathrm{~Hz}$, $p=32 \hbar k, \sigma_{z}=100 \mu \mathrm{m}$, and $k_{1}=2.01384 \times 10^{4} \mathrm{~m}^{-1}$.

choose $\xi=5.37$ since this provides optimal focusing in the $z=0$ plane.

The principle of BEC deposition using the variational method is illustrated in Figs. 2(a)-2(j). In the top row, the density profile of the released BEC in the $2 \mathrm{D}(x, z)$ plane is shown (the profile has been integrated over the $y$ axis). As it falls through the optical potential, it becomes more focused along the $x$ axis until it reaches its focal spot at $z=0$ [see Figs. 2(a)-2(d)]. It then begins to expand once it leaves the focal plane, $z=0$ [see Fig. 2(e)]. The second row [Figs. 2(f)-2(j)] shows the time integrated density in the $z=0$ plane, which we interpret as the accumulated or deposited density on the surface. As seen from left to right, this increases until all atoms have been deposited on the surface.

\section{RESULTS}

In this section, we conduct a number of numerical simulations using the variational approach and discuss the results. The effect of varying the potential power and imparted momentum kick to the BEC in the focusing event is studied for a variety of interatomic interactions. The advantages and drawbacks of using attractive BECs in high density regimes are investigated and we realize that in most cases, while utilizing an attractive BEC might provide a better profile resolution, it could also have detrimental impacts on the focused structures.

We assume an initial cylindrical BEC of $N_{0}=10^{5}{ }^{85} \mathrm{Rb}$ atoms confined by a harmonic trap of axial frequency $\omega_{0 x}=$ $2 \pi \times 10 \mathrm{~Hz}$ and radial frequency $\omega_{0 r}=\omega_{0 z}=\omega_{0 y}=2 \pi \times$ $70 \mathrm{~Hz}$. The center of the trap is located at $z_{0}=500 \mu \mathrm{m}$ from the center of the focusing harmonic potential. ${ }^{85} \mathrm{Rb}$ has an easily tuneable Feshbach resonance [57,58]. We initially set the $s$-wave scattering to $a_{s}=100 a_{0}\left(a_{0}\right.$ is the Bohr radius), giving a condensate with large mean-field energy and an inverted-parabolic Thomas-Fermi density profile. Once the trap is switched off and the BEC is allowed to freely propagate towards the focusing potential, we quench the scattering length to a value between $a_{s}=-10 a_{0}$ (attractive) and $a_{s}=100 a_{0}$ (repulsive). In the simplest case, the atoms are simply dropped from the trap and fall under gravity. We also consider scenarios where a momentum kick is imparted to the atoms upon release, which could be achieved with either a Raman or Bragg [59] optical transition. All results presented in this paper used a focusing potential with $\sigma_{z}=100 \mu \mathrm{m}, k_{1}=k / 400=2.01384 \times 10^{4} \mathrm{~m}^{-1}$ (where $k=$ $2 \pi / \lambda_{\mathrm{D}_{2}}=8.0554 \times 10^{6} \mathrm{~m}^{-1}$ ), and $\Delta=200 \mathrm{GHz}$, and use a three-body recombination loss rate coefficient of $K=4 \times$ $10^{-41} \mathrm{~m}^{6} \mathrm{~s}^{-1}$ [43].

Figure 3 shows the results of the integrated peak densities, FWHM, and atom number variation for different two-body interaction strengths, from $a_{s}=-1 a_{0}$ to $a_{s}=100 a_{0}$, when there are no momentum kicks. In this case, the required optimal power to focus the BEC at $z=0$ (center of the potential, the focal plane) is estimated as $P_{0 \hbar k}=4.127 \mathrm{~mW}$ by Eq. (26). For a comparison, results are also reproduced for different powers. We note that changing the field power for the same momentum kick (i.e., $p=0 \hbar k$ ) causes the focal plane to be shifted along the $z$ axis such that for $P>P_{0 \hbar k}$ and $P<P_{0 \hbar k}$, the focal plane is located, respectively, above and below the $z=0$ plane. Figure 4 represents the width dynamics of the BEC along the $x$ (transverse) axis as a function of the longitudinal position for four various powers: $0.5 P_{0 \hbar k}, P_{0 \hbar k}$, $2 P_{0 \hbar k}$, and $4 P_{0 \hbar k}$. Notice that for $P>P_{0 \hbar k}$, a breathinglike oscillation of the BEC occurs as it passes through the focusing potential along the $z$ axis. In such an event, multiple minimum peaks appear in the width dynamics. For $P=2 P_{0 \hbar k}$ (green dash-dotted curve) and $4 P_{0 \hbar k}$ (black dotted curve), the lowest minimum is $z_{f}=23.9 \mu \mathrm{m}$ and $z_{f}=44.3 \mu \mathrm{m}$, respectively, 

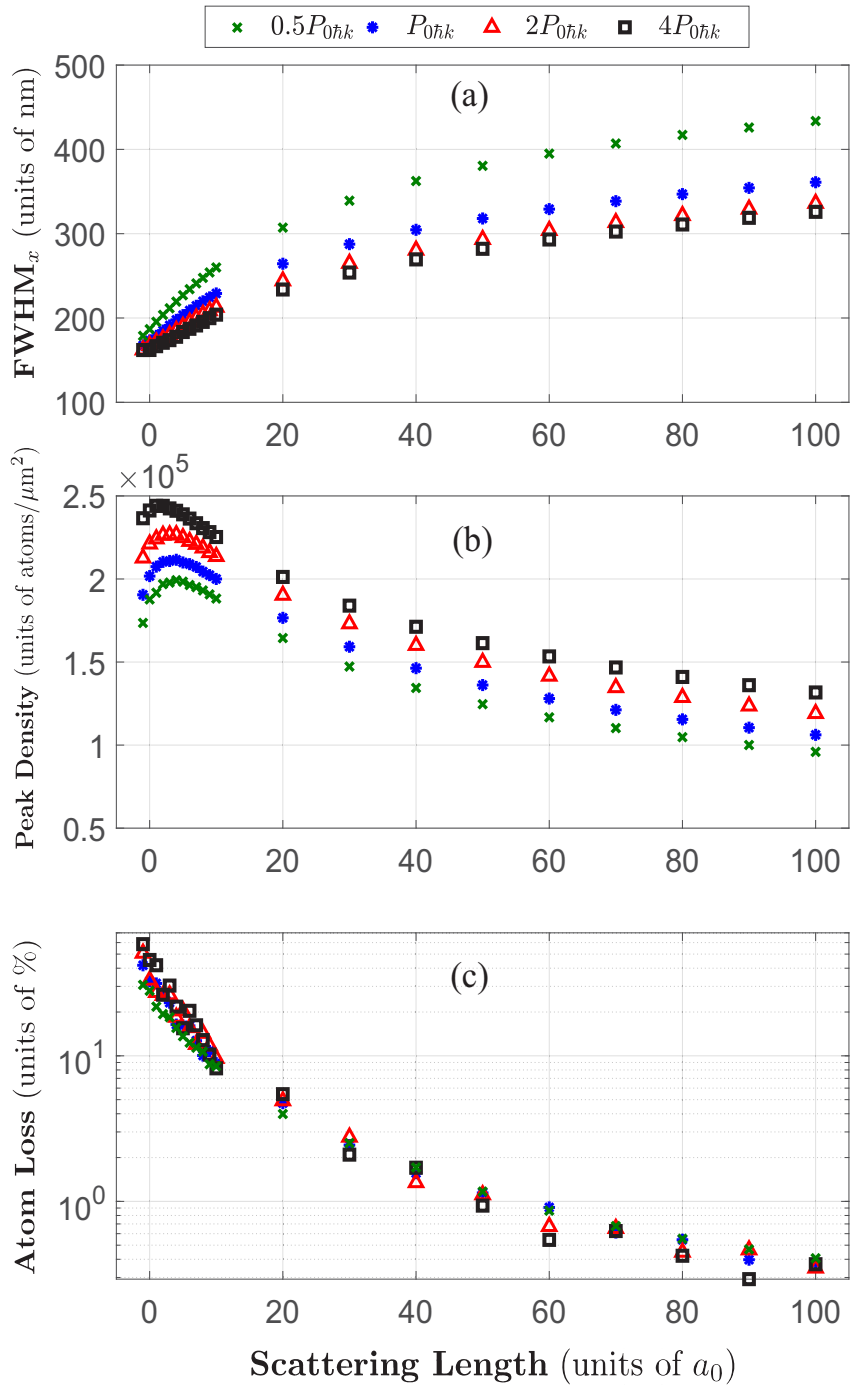

FIG. 3. Characterization of deposited 2D BEC density at different focal planes, determined from variational analysis for different values of scattering length and focusing power. (a)-(c) The values of FWHM, integrated peak densities, and atom loss due to three-body losses, respectively. The focal plane for $0.5 P_{0 \hbar k}, P_{0 \hbar k}$, $2 P_{0 \hbar k}$, and $4 P_{0 \hbar k}$ is, respectively, located at $z_{f}=-31.3,0,23.9$, and $44.3 \mu \mathrm{m}$ along the $z$ axis. Parameters used are $N_{0}=10^{5}$, $\sigma_{z}=100 \mu \mathrm{m}, k_{1}=2.01384 \times 10^{4} \mathrm{~m}^{-1}, \Delta=200 \mathrm{GHz}, I_{s}=16.7$ $\mathrm{W} / \mathrm{m}^{2}, \gamma=38 \mathrm{MHz}, v_{0}=0, v(z=0)=9.9 \mathrm{~cm} / \mathrm{s}, P_{0 \hbar k}=4.127$ $\mathrm{mW}, \mathcal{W}_{0 x}=19.6 \mu \mathrm{m}, \mathcal{W}_{0 z}=\mathcal{W}_{0 y}=2.8 \mu \mathrm{m}, \dot{\mathcal{W}}_{0 z}=\dot{\mathcal{W}}_{0 z}=\dot{\mathcal{W}}_{0 z}=$ $0, a_{0}=5.29 \times 10^{-11} \mathrm{~m}$, and $K=4 \times 10^{-41} \mathrm{~m}^{6} \mathrm{~s}^{-1}$.

occurring above the plane of $z=0$. For $P \leqslant P_{0 \hbar k}$, however, there always exists a single minimum peak, which is located at $z_{f}=0$ (red solid curve) and $-31.3 \mu \mathrm{m}$ (blue dashed curve) for $P_{0 \hbar k}$ and $0.5 P_{0 \hbar k}$, respectively. Hence, varying the power allows us to compare the quality of the deposited 2D BEC density at different focal planes; the results of this comparison are shown in Fig. 3.

As illustrated in Fig. 3(a), for a fixed power value, a decrease in the scattering length reduces the FWHM along the focusing $x$ direction. In other words, narrower and finer structures are given by smaller $s$-wave interactions. This variation becomes more significant as the BEC interactions switch from

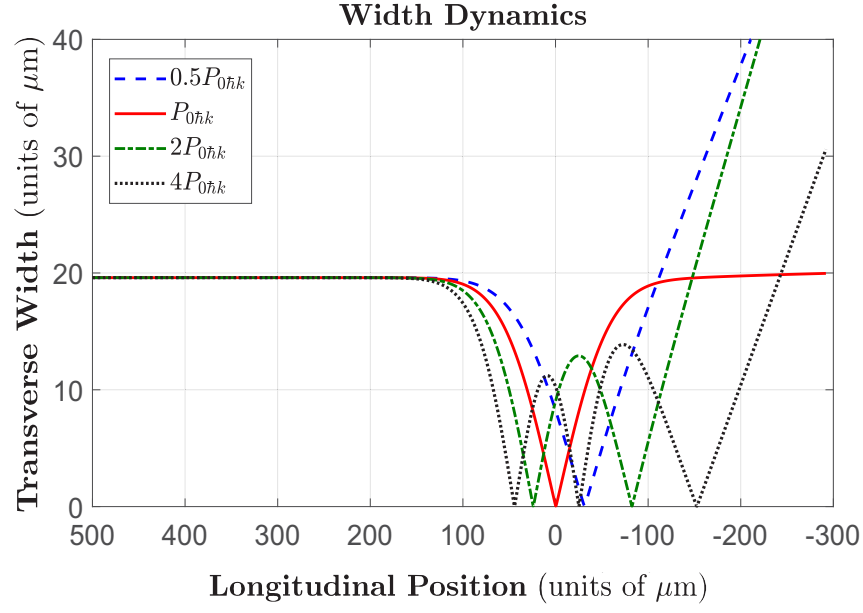

FIG. 4. Transverse width dynamics as a function of the longitudinal direction ( $z$ axis) for a focusing BEC dropped at $z_{0}=500 \mu \mathrm{m}$ with zero momentum kick, $p=0 \hbar k$. The blue dashed, red solid, green dash-dotted, and black dotted curves, respectively, correspond to $0.5 P_{0 \hbar k}, P_{0 \hbar k}, 2 P_{0 \hbar k}$, and $4 P_{0 \hbar k}$, where $P_{0 \hbar k}$ is the optimal power to focus the BEC at $z=0$ and is estimated as $4.127 \mathrm{~mW}$. The lowest minimum peak (the focal plane) for $0.5 P_{0 \hbar k}, P_{0 \hbar k}, 2 P_{0 \hbar k}$, and $4 P_{0 \hbar k}$ is, respectively, $z_{f}=-31.3,0,23.9$, and $44.3 \mu \mathrm{m}$. Parameters are $N_{0}=$ $10^{5}, \sigma_{z}=100 \mu \mathrm{m}, k_{1}=2.01384 \times 10^{4} \mathrm{~m}^{-1}, \Delta=200 \mathrm{GHz}, I_{s}=$ $16.7 \mathrm{~W} / \mathrm{m}^{2}, \gamma=38 \mathrm{MHz}, \mathcal{W}_{0 x}=19.6 \mu \mathrm{m}, \mathcal{W}_{0 z}=\mathcal{W}_{0 y}=2.8 \mu \mathrm{m}$, $a_{s}=100 a_{0}, a_{0}=5.29 \times 10^{-11} \mathrm{~m}$, and $K=4 \times 10^{-41} \mathrm{~m}^{6} \mathrm{~s}^{-1}$.

repulsive to attractive. For $a_{s}>5 a_{0}$, decreasing the scattering length results in higher peak densities since repulsive interactions hinder the focusing of the atoms. However, for $a_{s} \leqslant 5 a_{0}$, lowering $a_{s}$ causes the peak densities to decrease; see Fig. 3(b). This is due to the three-body recombination losses which become increasingly important as the density of the condensate increases. This explains the loss rate of atoms in Fig. 3(c), which increases as the $s$-wave scattering length is reduced. Although three-body losses minimally affect a BEC with large repulsive interactions, they become significant in higher density regimes for $a_{s} \leqslant 10 a_{0}$, where up to $60 \%$ of the atoms can be lost. Altering the laser power also substantially affects the deposition. According to Figs. 3(a)-3(c), increasing the power leads to a better resolution (smaller FWHM) and larger peak density for each value of $a_{s}$, at the expense of moving the focal plane.

We now investigate in more detail the effect of attractive interactions for $-10 a_{0} \leqslant a_{s} \leqslant-1 a_{0}$. In this regime, small decreases in the value of the scattering length can considerably alter the density profile. Figure 5(a) compares the BEC widths, along all $x, y$, and $z$ axes for different scattering lengths $a_{s}=-10,-5,-1 a_{0}$, as a function of longitudinal position, $z$. In this figure, the BEC is prepared at $z_{0}=500 \mu \mathrm{m}$ and dropped (no momentum kick) at $t=0$. The minimum possible value for $\mathcal{W}_{x}$ for all three interaction strengths almost occurs at $z=0$ (see the overlap between the blue solid, dashed, and dash-dotted curves). However, the resultant radial widths, $\mathcal{W}_{y}$ and $\mathcal{W}_{z}$, are quite different from one scattering length to another (see the red solid, dashed, and dash-dotted curves). As revealed, the minimum peak in the $\mathcal{W}_{y}$ and $\mathcal{W}_{z}$ curves is shifted closer to $z=0$ as $a_{s}$ is reduced such that for $a_{s}=-10 a_{0}$, this point takes place before $z=0(z<0)$. 


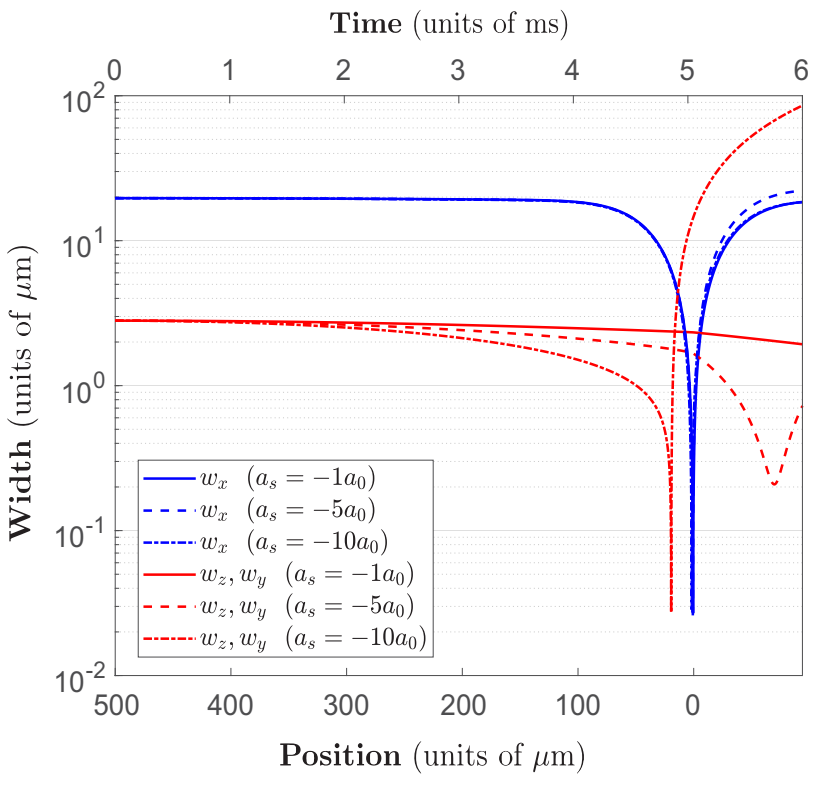

(a)

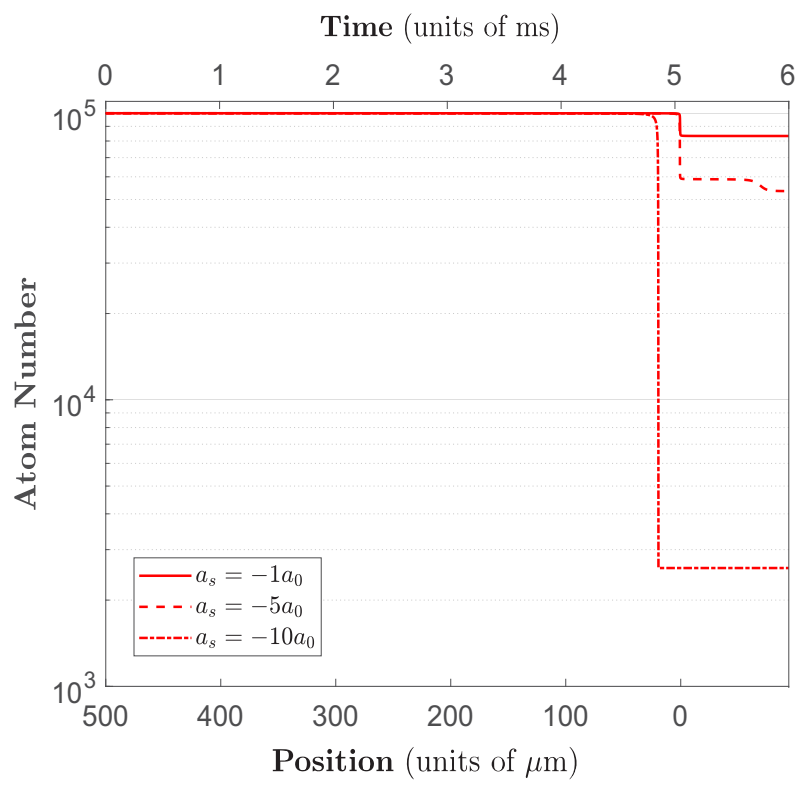

(b)

FIG. 5. (a) The width dynamics for $a_{s}=-10 a_{0}$ (dash-dotted curves), $-5 a_{0}$ (dashed curves), and $-1 a_{0}$ (solid curves), for a BEC optimally focused at $z=z_{f}=0$. The blue curves illustrate the BEC width evolution along the $x$ axis, whereas the red curves represent this trend along the $y$ and $z$ axes. (b) The corresponding atom number evolution for the same three scattering lengths. Parameters are $N_{0}=10^{5}, z_{0}=500 \mu \mathrm{m}, \sigma_{z}=$ $100 \mu \mathrm{m}, k_{1}=2.01384 \times 10^{4} \mathrm{~m}^{-1}, \Delta=200 \mathrm{GHz}, \mathcal{W}_{0 x}=19.6 \mu \mathrm{m}, \mathcal{W}_{0 z}=\mathcal{W}_{0 y}=2.8 \mu \mathrm{m}, a_{0}=5.29 \times 10^{-11} \mathrm{~m}$, and $K=4 \times 10^{-41} \mathrm{~m}^{6} \mathrm{~s}^{-1}$.

This effect is well explained by the collapse of a BEC in high density regimes $[44,60,61]$ where the attractive interactions between atoms cause the BEC to rapidly and strongly shrink at a critical density and then to expand sharply. As shown in Fig. 5(b), as the BEC collapses, there is consequently a significant loss of atoms due to three-body recombination losses.

In Fig. 6, we examine the impact of initial momentum kicks on the FWHM, peak densities, and atom loss. Each momentum kick requires a particular optimal power since higher momentum kicks require larger powers to bring atoms to the same spot. For example, for a kick towards the focal plane with a magnitude of $2 \hbar k$, the corresponding optimal power of $P_{2 \hbar k}=4.188 \mathrm{~mW}$ is required to focus at $z=z_{f}=0$. For larger momentum kicks, the powers needed for a focal plane of $z_{f}=0$ are $\left(P_{4 \hbar k}, P_{6 \hbar k}, P_{8 \hbar k}, P_{10 \hbar k}, P_{12 \hbar k}, P_{14 \hbar k}, P_{16 \hbar k}\right)=$ $(4.372,4.678,5.106,5.656,6.329,7.124,8.041) \mathrm{mW}$. As indicated in Fig. 6(a), more powerful kicks reduce the widths of the deposited atoms along the $x$ direction. Increasing the momentum kick leads to slightly higher peak densities for repulsive BECs in regimes where the three-body recombination effects are insignificant [see Fig. 6(c)]. However, larger kicks give smaller peak densities for a small negative $a_{s}$, as seen in Fig. 6(b) for $a_{s}=-1 a_{0}$.

Finally, in addition to the study of the accumulative atomic flux on a substrate during the focus process, it is also worth considering the resultant profile right at the moment of optimal focus at $z=z_{f}=0$, known as an instantaneous profile. This has been used in previous theoretical work to examine the structures predicted by the particle optics or classical trajectories approach [14]. Figures 7(a)-7(c) illustrate the related outcomes of resolution, peak density, and atom loss for a propagating condensate immediately at the time its center of mass reaches the center of the focusing plane, $z=0$. As above, it is assumed that the condensate has started its free propagation at $z_{0}=500 \mu \mathrm{m}$ and is exposed to a focusing potential comprising the parameters of $k_{1}=2.01384 \times 10^{4} \mathrm{~m}^{-1}, \sigma_{z}=$ $100 \mu \mathrm{m}$, and $\Delta=200 \mathrm{GHz}$. There appears a more rapid trend in the FWHM over different ranges of scattering length [see Fig. 7(a)] when a momentum kick of $p=32 \hbar k$ is applied compared to that of $p=64 \hbar k$ and $p=128 \hbar k$. The impact of increasing the scattering length on the structure resolution is more significant for lower kicks, especially in the regime of relatively low $a_{s}$ where the three-body losses are non-negligible. This is also the case for the scattering length dependence of the peak density [see Fig. 7(b)]. Although the trend remains steady with a gradual slope for $p=128 \hbar k$, it undergoes a fluctuation around $a_{s} \sim 1 a_{0}$ for lower kicks (i.e., $p=64 \hbar k)$, which becomes steeper with a decrease in kick values as seen for $p=32 \hbar k$. Turning to the atom number loss shown in Fig. 7(c), the amount of loss in high density regimes for lower kicks (e.g., $p=32 \hbar k$ ) is considerably greater than higher kicks. In fact, the BEC with a slower longitudinal velocity is exposed to the field for a relatively longer time, resulting in a larger atom loss, whereas for higher velocities (i.e., $p=128 \hbar k$ ), the BEC has less chance to interact with the field. Of the parameters that we considered, $a_{s}=-1 a_{0}$ and $p=128 \hbar k$ gave the instantaneous profile with the best resolution $\left(\mathrm{FWHM}_{x} \simeq 9 \mathrm{~nm}\right)$ and highest peak density $\left(2 \times 10^{6}\right.$ atoms $\left./ \mu \mathrm{m}^{2}\right)$.

It should be noted that in terms of the three-body recombination effect, the variational methodology only scales the impact of the ejected atoms from the BEC (consequently, from the harmonic potential depth) on the profile structures. Given that most of the loss occurs at or close to the surface, many of the lost atoms may still be deposited on the surface, leading to some smearing around the pedestal of the density profile. 

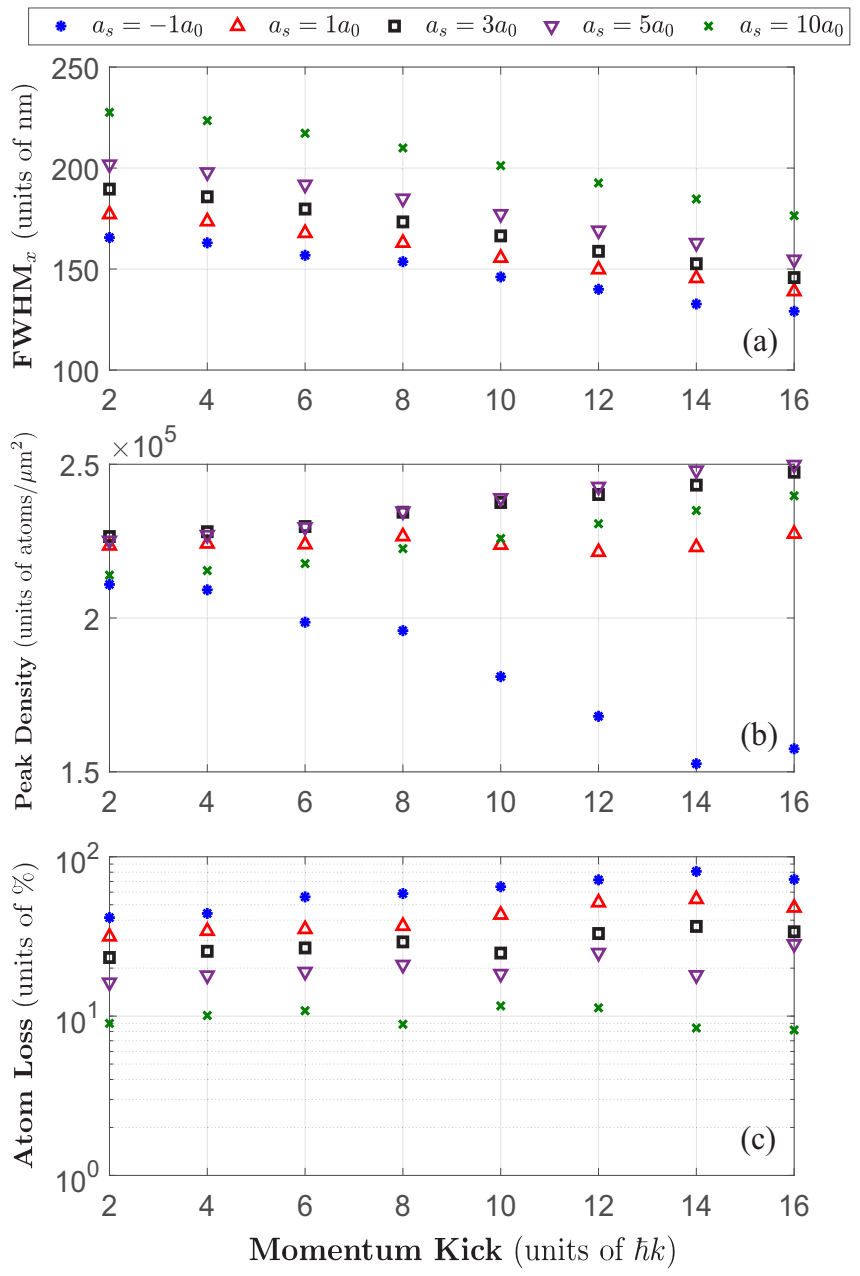

FIG. 6. Characterization of deposited 2D BEC density at $z=$ 0 , determined from the variational analysis for different momentum kicks and scattering lengths. (a)-(c) The values of FWHM, integrated peak density, and the amount of atom loss, respectively. Parameters are $N_{0}=10^{5}, z_{0}=500 \mu \mathrm{m}, \sigma_{z}=100 \mu \mathrm{m}, k_{1}=$ $2.01384 \times 10^{4} \mathrm{~m}^{-1}, \Delta=200 \mathrm{GHz}, I_{s}=16.7 \mathrm{~W} / \mathrm{m}^{2}, \gamma=38 \mathrm{MHz}$, $\mathcal{W}_{0 x}=19.6 \mu \mathrm{m}, \quad \mathcal{W}_{0 z}=\mathcal{W}_{0 y}=2.8 \mu \mathrm{m}, \quad \dot{\mathcal{W}}_{0 z}=\dot{\mathcal{W}}_{0 z}=\dot{\mathcal{W}}_{0 z}=0$, $a_{0}=5.29 \times 10^{-11} \mathrm{~m}$, and $K=4 \times 10^{-41} \mathrm{~m}^{6} \mathrm{~s}^{-1}$ for Rb 85 .

\section{NUMERICAL SIMULATIONS (GPE)}

In order to investigate the accuracy of our variational solutions, we compare with GPE numerical simulations. We numerically solved Eq. (1) with an embedded Runge-Kutta (ERK) scheme along with adaptive Fourier split-step size [62]. We used the third and fourth orders [ERK4(3)] to deliver an estimation of the local error for adaptive step-size control purposes in the interaction picture. The initial condition for each simulation was the GPE ground state, numerically determined via imaginary-time evolution. Our simulations indicate that the variational method predicts the evolution of the BEC widths and density functions well. Figure 8 represents the top view of the deposited profile on the surface $z=0$ extracted by both the numerical GPE and variational approach when $a_{s}=$ $-1 a_{0}$ and with zero momentum kick. The GPE results for a variety of $s$-wave scattering lengths, when the potential power is set to $P_{0 \hbar k}$, are shown in Fig. 9 as well as the corresponding
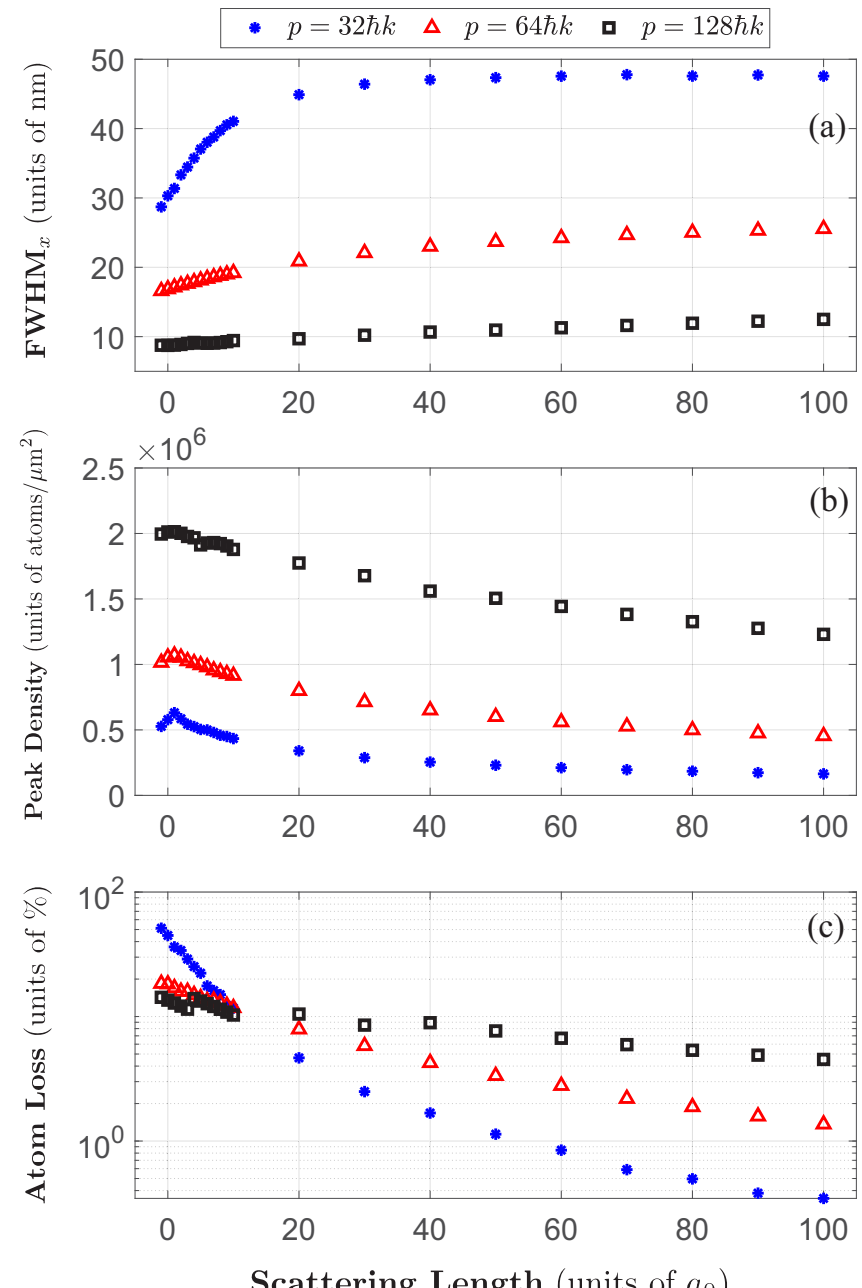

FIG. 7. The simulation results of the instantaneous focused profile at $z=0$ when no substrate is considered. The results are determined from the variational method for different momentum kicks and scattering lengths. (a)-(c) The values of FWHM, integrated peak density, and the amount of atom loss, respectively. Parameters are $N_{0}=10^{5}, z_{0}=500 \mu \mathrm{m}, \sigma_{z}=100 \mu \mathrm{m}, k_{1}=$ $2.01384 \times 10^{4} \mathrm{~m}^{-1}, \Delta=200 \mathrm{GHz}, I_{s}=16.7 \mathrm{~W} / \mathrm{m}^{2}, \gamma=38 \mathrm{MHz}$, $\mathcal{W}_{0 x}=19.6 \mu \mathrm{m}, \quad \mathcal{W}_{0 z}=\mathcal{W}_{0 y}=2.8 \mu \mathrm{m}, \quad \dot{\mathcal{W}}_{0 z}=\dot{\mathcal{W}}_{0 z}=\dot{\mathcal{W}}_{0 z}=0$, $a_{0}=5.29 \times 10^{-11} \mathrm{~m}$, and $K=4 \times 10^{-41} \mathrm{~m}^{6} \mathrm{~s}^{-1}$ for Rb 85 .

variational calculations. There is a good agreement between the two approaches, especially in the interval of $a_{s} \leqslant 10 a_{0}$.

It is worth noting that for a BEC moving relatively slowly through the focusing potential, the dimensionality disruptions observed in the GPE simulations, which result from excitations, are negligible. Hence, in the slow regime, one can expect reasonable agreement between the focused profile distributions of the GPE and variational approaches. However, as higher momentum kicks are applied to the BEC, excitations during the focusing process become more significant. Figure 10 illustrates this by comparing the BEC density profiles along the $x$ axis as calculated from the variational approach and GPE simulations. For $a_{s}=100 a_{0}$ and $p=32 \hbar k$, the GPE simulations show that significant fringes emerge, which are not captured in the variational methodology. The estimated resolutions are still in a close agreement, $(\Delta x)_{\mathrm{var}}^{32 \hbar k}=$ 


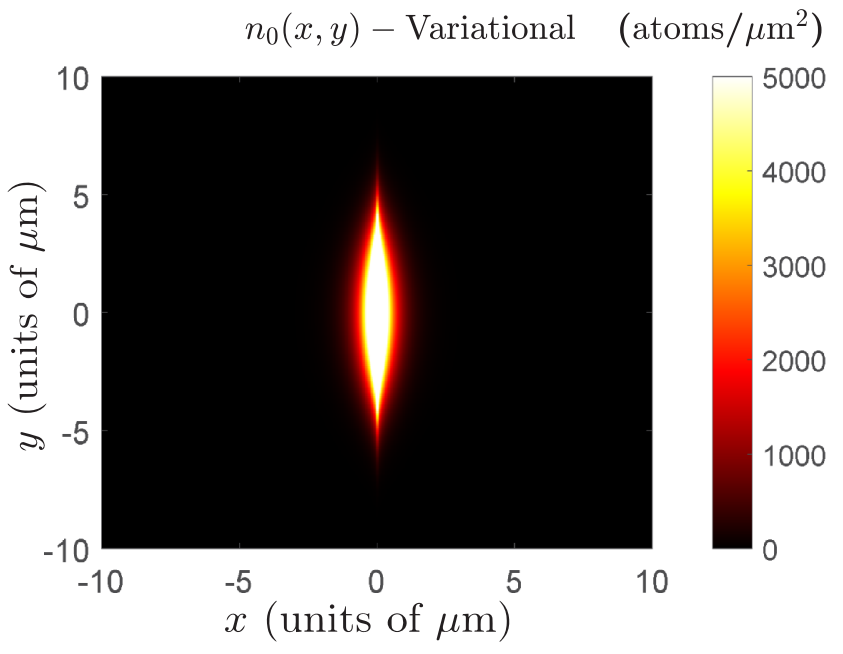

(a)

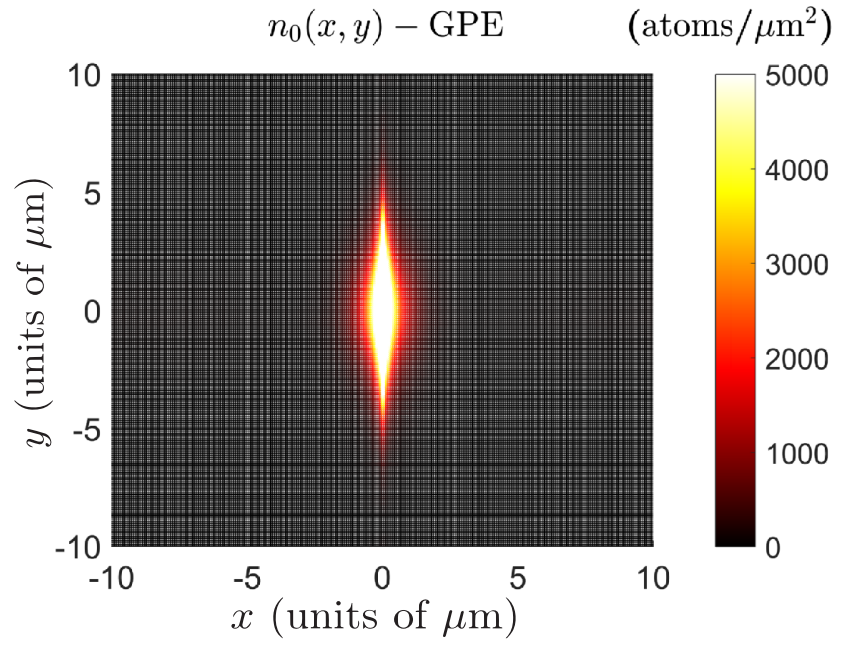

(b)

FIG. 8. (a) Deposited BEC focused structure on the $(x, y)$ plane $(z=0)$ carried out via the variational approach. (b) Deposited BEC profile on the same plane acquired by a numerical GPE simulation. In both simulations, no momentum kick is applied to the BEC and $a_{s}=-1 a_{0}$. Parameters are $N_{0}=10^{5}, \omega_{0 x}=2 \pi \times 10 \mathrm{~Hz}, \omega_{0 y}=\omega_{0 z}=2 \pi \times 70 \mathrm{~Hz}, p=32 \hbar k, \sigma_{z}=100 \mu \mathrm{m}$, and $k_{1}=2.01384 \times 10^{4} \mathrm{~m}^{-1}$.

$47.5 \mathrm{~nm}$ and $(\Delta x)_{\mathrm{GPE}}^{32 \hbar k}=46.4 \mathrm{~nm}$. In the case of no momentum kick, both the GPE and variational distributions tend to a Gaussian profile (although the GPE curve has a longer tail than the variational one; see the solid green and dashed red curves). The tail in the GPE simulations reduces by increasing the momentum kick, which necessitates an increase in the potential power and intensity accordingly for the same focus spot. Again, the estimated resolutions are in close agreement with $(\Delta x)_{\mathrm{var}}^{0, k}=130.1 \mathrm{~nm}$ and $(\Delta x)_{\mathrm{GPE}}^{0, \hbar k}=133.9 \mathrm{~nm}$.

\section{CONCLUSION}

We have investigated the focusing dynamics of $\mathrm{a}^{85} \mathrm{Rb} \mathrm{BEC}$ with two-body interactions and three-body recombination losses due to a harmonic-shaped focusing optical potential. Using a variational technique, we derived the dynamical behavior of the peak densities, FWHMs, and atom number loss in the focusing regimes when tuning the scattering length over a large range. We showed that the interparticle interaction can play an essential role in the deposited profile structures, which become more sensitive to smaller $a_{s}$ as well as its negative values. We conclude that high peak densities and small resolutions can be achieved in relatively attractive BECs. However, the three-body losses limit the maximum achievable density and, when sufficient, can disrupt the deposition quality. Since highly repulsive BECs are exposed to negligible amounts of atom loss in the focusing regime, applying a momentum kick to the BEC can always be beneficial to both the peak density and resolution of focused structures. Nevertheless, the scenario is completely different for the attractive BECs. In this case, although higher momentum kicks and powers may provide one with more resolution transparency, they can still destroy the created peak densities. Last but not least, we inferred that to improve the profile resolution at the same focal plane, one needs to boost the momentum kick in accordance with the potential power, whereas for the same momentum kick, increasing the power leads to a focal plane displacement; this is optimally shifted along the longitudinal axis to an area above the center of the focusing potential.

It is worth mentioning that examining focusing using a one-dimensional (1D) light field is a good proof of principle and the fastest way to realize the realistic atom lithography, such as considering the influence of varying different light field parameters, as well as various momentum kicks and interaction strengths on the focused profile. While using a two-dimensional standing wave [63] (i.e., 2D lattice) as a 2D light mask results in square arrays of fabricated structures, there is a serious challenge in the experimental setup with a 2D light mask: the deposited pattern is strongly dependent on the relative temporal phase between the two Gaussian waves. In this case, the pattern can easily be disrupted if the temporal phase is not aligned (stabilized) appropriately. Moreover, using a 2D focusing light, almost no improvement is achieved to the profile resolution, and the profile linewidth along the $x$ and $y$ directions would approximately be the same at the end of focusing. This implies that adding the transverse $y$ axis in the focusing event would not influence the physics of the problem in atom deposition. However, utilizing a 2D light mask in focusing BECs along with a fair discussion of the challenges associated with this could remain as possibilities for future work.

We also note that the quantum reflection from the surface has been neglected in our model. This is strongly dependent on the longitudinal velocity of the falling BEC, which becomes important for relatively low kinetic energies and longitudinal velocities. In [64], it has been shown that the quantum reflection in the presence of interatomic interactions becomes significant for the atom cloud when $v \leqslant 2 \mathrm{~mm} / \mathrm{s}$. In our simulations, the lowest value of the initial momentum kick used is $p=0 \hbar k$, resulting in $v=9.9 \mathrm{~cm} / \mathrm{s}$ (see Fig. 3) at the surface, which is much larger than the critical value of quantum reflection regimes [64].

Overall, we conclude that reaching nanometer-scale structures using a BEC source is achievable, which provides much 

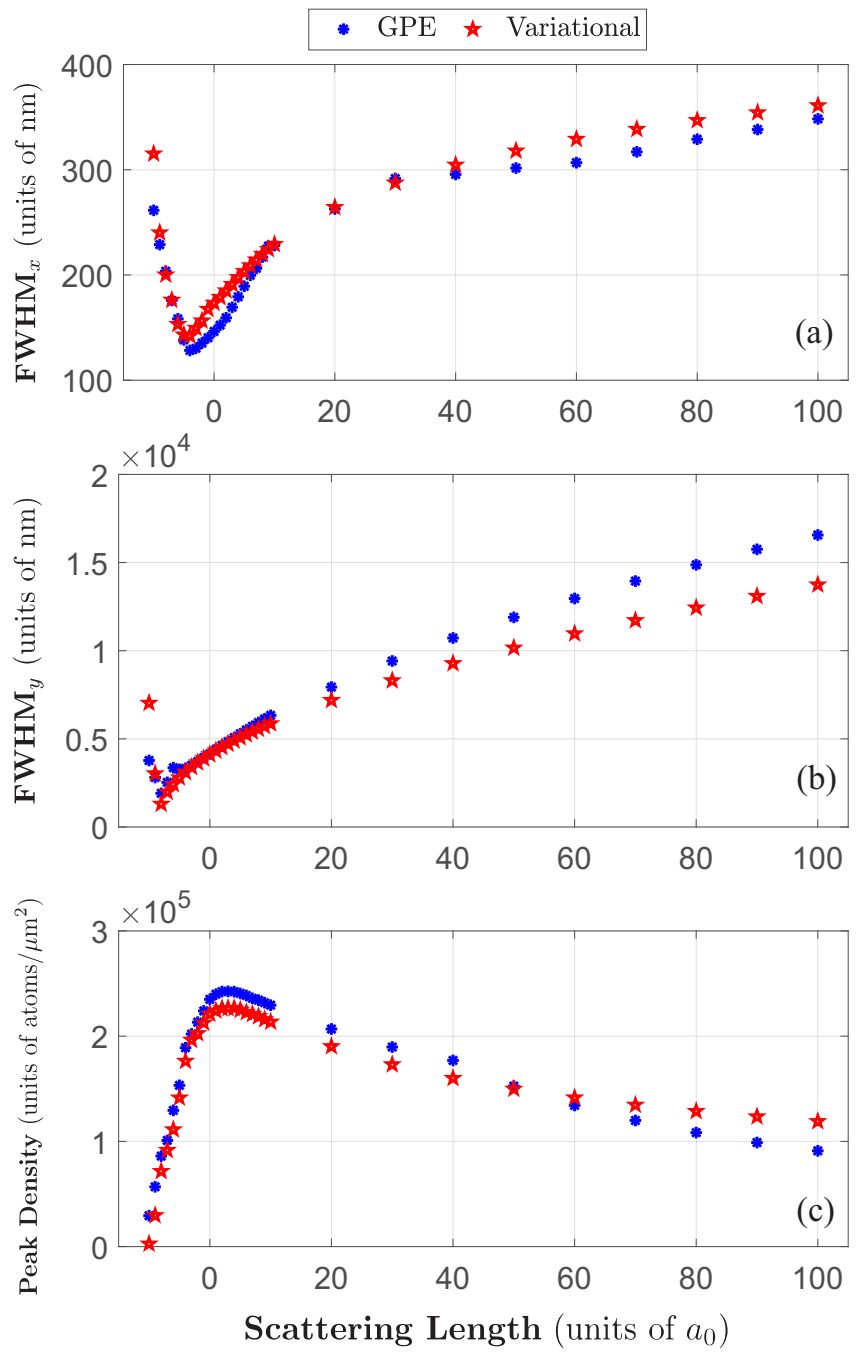

FIG. 9. (a) The values of FWHM of the focused distribution along the $x$ axis for different $a_{s}$. (b) The FWHMs along one of the radial axes, $y$, against various scattering lengths. (c) The achieved values of peak densities of the focused profile when using different interaction strengths. The red dots in all three graphs indicate predictions from the variational method, whereas the blue dots show results from the numerical GPE simulations. Parameters are $N_{0}=10^{5}, z_{0}=$ $500 \mu \mathrm{m}, \sigma_{z}=100 \mu \mathrm{m}, k_{1}=2.01384 \times 10^{4} \mathrm{~m}^{-1}, \Delta=200 \mathrm{GHz}$, $I_{s}=16.7 \mathrm{~W} / \mathrm{m}^{2}, \gamma=38 \mathrm{MHz}, p=0 \hbar k, P_{0 \hbar k}=4.127 \mathrm{~mW}, a_{0}=$ $5.29 \times 10^{-11} \mathrm{~m}$, and $K=4 \times 10^{-41} \mathrm{~m}^{6} \mathrm{~s}^{-1}$.

higher profile resolution and peak density than those created by thermal atomic sources. Finally, we have demonstrated the power of using a variational methodology to examine a broad range of parameter space which would not be easily achievable using 3D GPE simulations.

\section{ACKNOWLEDGMENTS}

The authors would like to thank Nicholas P. Robins and Hans A. Bachor for useful discussions and feedback. Grateful acknowledgment is also extended to Timothy Senden for the financial support of the project. S.S.S. was supported by an Australian Research Council Discovery Early Career Researcher Award (DECRA), Project No. DE200100445.

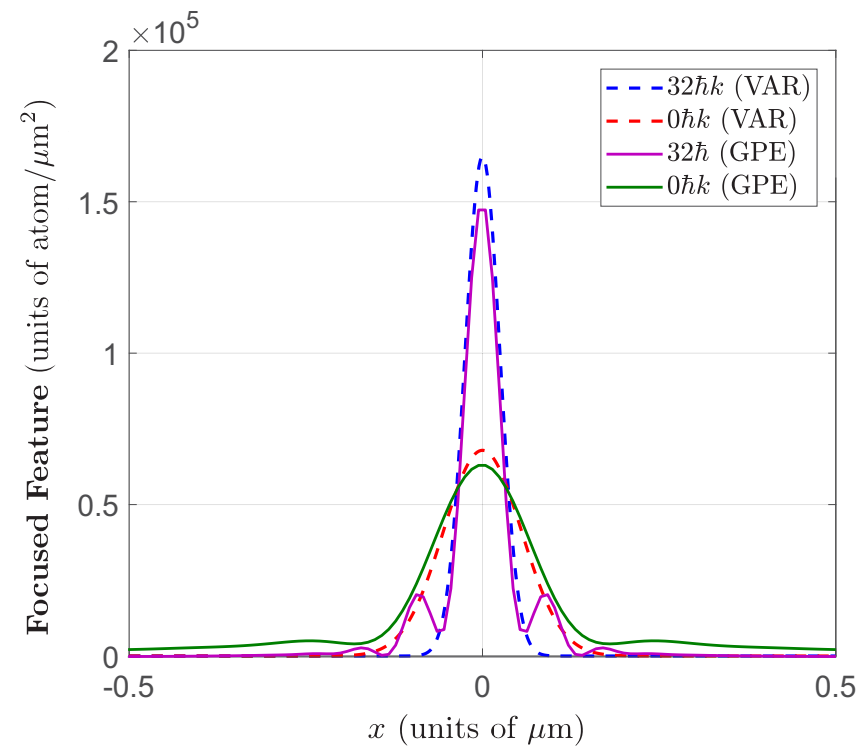

FIG. 10. BEC focused structures $n_{0}(x)$ along the transverse $x$ axis on the planes $y=0$ and $z=0$ achieved via the variational approach (dashed curves) and GPE simulations (solid curves) for $p=0$ (green and red curves) and $32 \hbar k$ (blue and purple curves). The BEC scattering length is set to $100 a_{0}$ in all the simulations.

\section{APPENDIX}

Here we provide a detailed description of our analytical methods, including a derivation of the Lagrangian given by Eq. (13) and the variational equations (17)-(20).

\section{Derivation of Lagrangian function given by Eq. (13)}

Substituting Eq. (11) into Eq. (8) and simplifying give the Lagrangian density,

$$
\begin{aligned}
\mathcal{L}(\mathbf{r}, t)= & -\sum_{r=x, y, z}|A|^{2} \exp \left(-r^{2} / \mathcal{W}_{r}^{2}\right) \\
& \times\left\{\hbar\left[\dot{\beta}_{r} r^{2}+\dot{\alpha}_{r} r+\dot{\phi}_{r}\right]+\frac{\hbar^{2}}{2 m}\left[\frac{r^{2}}{\mathcal{W}_{r}^{4}}+4 \beta_{r}^{2} r^{2}\right.\right. \\
& \left.\left.+4 \beta_{r} \alpha_{r} r+\alpha_{r}^{2}\right]-\frac{1}{2}|A|^{2} u\right\} \\
& -\frac{1}{2} m|A|^{2} \omega_{x}^{2}(t) x^{2} \exp \left(-x^{2} / \mathcal{W}_{x}^{2}\right)
\end{aligned}
$$

Note that this excludes the three-body recombination loss term. Inserting Eq. (A1) into $L(t)=\int d^{3} \mathbf{r} \mathcal{L}(\mathbf{r}, t)$ gives the Lagrangian

$$
\begin{aligned}
L(t)= & -\sum_{r=x, y, z} \frac{|A|^{2} \mathcal{W}_{r} \sqrt{\pi}}{2}\left\{\hbar \dot{\beta}_{r} \mathcal{W}_{r}^{2}+2 \hbar \dot{\phi}_{r}+\frac{\hbar^{2}}{2 m}\left(\frac{1}{\mathcal{W}_{r}^{2}}\right)\right. \\
& \left.+\frac{\hbar^{2}}{2 m}\left(4 \beta_{r}^{2} \mathcal{W}_{r}^{2}\right)+\frac{\hbar^{2}}{2 m}\left(2 \alpha_{r}^{2}\right)+\frac{1}{2} m \omega_{x}^{2}(t) \mathcal{W}_{x}^{2}+\frac{g|A|^{2}}{\sqrt{2}}\right\},
\end{aligned}
$$


where the following three well-known Gaussian integrals have been used:

$$
\begin{aligned}
& \int_{-\infty}^{\infty} \exp \left(-a r^{2}\right) d r=\sqrt{\frac{\pi}{a}}, \\
& \int_{-\infty}^{\infty} r \exp \left(-a r^{2}\right) d r=0, \\
& \int_{-\infty}^{\infty} r^{2} \exp \left(-a r^{2}\right) d r=\frac{\sqrt{\pi}}{2 a^{3 / 2}},
\end{aligned}
$$

where $a$ is a constant.

\section{Derivation of BEC variational equations}

Substituting Eq. (13) into Eq. (16) and solving for $q_{i}=$ $\mathcal{A}, \mathcal{W}_{r}, \alpha_{r}, \beta_{r}, \phi_{r}$ gives the following respective equations:

$$
\begin{gathered}
\sum_{r=x, y, z}\left\{\frac{\hbar \dot{\beta}_{r} \mathcal{W}_{r}^{2}}{2}+\hbar \dot{\phi}_{r}+\frac{\hbar^{2}}{m}\left(\frac{1}{4 \mathcal{W}_{r}^{2}}\right)+\frac{\hbar^{2}}{m}\left(\beta_{r}^{2} \mathcal{W}_{r}^{2}\right)\right. \\
\left.+\frac{\hbar^{2}}{2 m} \alpha_{r}^{2}+\frac{g|A|^{2}}{\sqrt{2}}\right\}+\frac{1}{4} m \omega_{x}^{2}(t) \mathcal{W}_{x}^{2}=0, \\
\sum_{r=x, y, z}\left\{\frac{3}{2} \hbar \dot{\beta}_{r} \mathcal{W}_{r}^{2}+\hbar \dot{\phi}_{r}-\frac{\hbar^{2}}{m}\left(\frac{1}{4 \mathcal{W}_{r}^{2}}\right)+\frac{\hbar^{2}}{m}\left(3 \beta_{r}^{2} \mathcal{W}_{r}^{2}\right)\right. \\
\left.+\frac{\hbar^{2}}{2 m} \alpha_{r}^{2}+\frac{g|A|^{2}}{2 \sqrt{2}}\right\}+\frac{3}{4} m \omega_{x}^{2}(t) \mathcal{W}_{x}^{2}=0, \\
\sum_{r=x, y, z}\left\{\frac{\hbar^{2}}{m}|A|^{2} \mathcal{W}_{r} \alpha_{r}\right\}=0, \\
\left\{\hbar \frac{d}{d t}\left(|A|^{2} \mathcal{W}_{r}^{3}\right)-\frac{\hbar^{2}}{m}\left(4|A|^{2} \beta_{r} \mathcal{W}_{r}^{3}\right)+\frac{K|A|^{6} \mathcal{W}_{r}^{3}}{9 \sqrt{3}}\right\}=0, \\
\sum_{r=x, y, z}\left\{\hbar \frac{d}{d t}\left(|A|^{2} \mathcal{W} \mathcal{W}_{r}\right)+\frac{K|A|^{6} \mathcal{W}_{r}}{3 \sqrt{3}}\right\}=0 .
\end{gathered}
$$

According to the normalization condition $N(t)=$ $\int_{-\infty}^{+\infty} \psi \psi^{*} d r$ and Eq. (11), we can write

$$
\frac{d N(t)}{d t}=\sqrt{\pi} \frac{d}{d t}\left(|A|^{2} \mathcal{W}_{r}\right)
$$

Combining Eqs. (A7) and (A8), and using Eq. (12), gives the associated differential equation for the change of atom number in the condensate,

$$
\frac{d N(t)}{d t}=-\frac{K N^{3}}{3 \sqrt{3} \hbar \pi \mathcal{W}_{r}^{2}} .
$$

We then merge Eq. (A4) and Eq. (A5) to acquire

$$
\begin{aligned}
& \sum_{r=x, y, z}\left\{\hbar \dot{\beta}_{r} \mathcal{W}_{r}^{2}+\frac{\hbar^{2}}{m}\left(2 \beta_{r}^{2} \mathcal{W}_{r}^{2}-\frac{1}{2 \mathcal{W}_{r}^{2}}\right)-\frac{g|A|^{2}}{2 \sqrt{2}}\right\} \\
& +\frac{1}{2} m \omega_{x}^{2}(t) \mathcal{W}_{x}^{2}=0 .
\end{aligned}
$$

Considering the amplitude $|A|$ via Eq. (12); this implies that

$$
\begin{gathered}
\dot{\beta}_{x}=\left(\frac{\hbar}{2 m}\right) \frac{1}{\mathcal{W}_{x}^{4}}-\left(\frac{2 \hbar}{m}\right) \beta_{x}^{2}-\left(\frac{m}{2 \hbar}\right) \omega_{x}^{2}(t) \\
+\frac{g N}{2(2 \pi)^{3 / 2} \hbar \mathcal{W}_{x}^{3} \mathcal{W}_{y} \mathcal{W}_{z}}, \\
\dot{\beta}_{y}=\left(\frac{\hbar}{2 m}\right) \frac{1}{\mathcal{W}_{y}^{4}}-\left(\frac{2 \hbar}{m}\right) \beta_{y}^{2}+\frac{g N}{2(2 \pi)^{3 / 2} \hbar \mathcal{W}_{x} \mathcal{W}_{y}^{3} \mathcal{W}_{z}}, \\
\dot{\beta}_{z}=\left(\frac{\hbar}{2 m}\right) \frac{1}{\mathcal{W}_{z}^{4}}-\left(\frac{2 \hbar}{m}\right) \beta_{z}^{2}+\frac{g N}{2(2 \pi)^{3 / 2} \hbar \mathcal{W}_{x} \mathcal{W}_{y} \mathcal{W}_{z}^{3}} .
\end{gathered}
$$

Similarly, mixing Eq. (A7) and Eq. (A8) gives

$$
\sum_{r=x, y, z}\left\{\hbar \dot{\mathcal{W}}_{r}-\frac{2 \hbar^{2}}{m}\left(\beta_{r} \mathcal{W}_{r}\right)-\frac{K|A|^{4} \mathcal{W}_{r}}{9 \sqrt{3}}\right\}=0
$$

Again, using Eq. (12), this gives the evolution of the $x$ component width,

$$
\dot{\mathcal{W}}_{x}=\frac{2 \hbar}{m}\left(\beta_{x} \mathcal{W}_{x}\right)+\frac{K N^{2}}{\sqrt{3}(3 \pi)^{3} \hbar \mathcal{W}_{x} \mathcal{W}_{y}^{2} \mathcal{W}_{z}^{2}}
$$

Taking the second derivative of (A16) with respect to time for $x$-dependent variables gives

$$
\begin{aligned}
\ddot{\mathcal{W}}_{x}= & \frac{2 \hbar}{m}\left(\dot{\beta}_{x} \mathcal{W}_{x}+\beta_{x} \dot{\mathcal{W}}_{x}\right)+\frac{2 \dot{N} N K}{\sqrt{3}(3 \pi)^{3} \hbar \mathcal{W}_{x} \mathcal{W}_{y}^{2} \mathcal{W}_{z}^{2}} \\
& -\frac{K N^{2} \dot{\mathcal{W}}_{x}}{\sqrt{3}(3 \pi)^{3} \hbar \mathcal{W}_{x}^{2} \mathcal{W}_{y}^{2} \mathcal{W}_{z}^{2}} .
\end{aligned}
$$

Using the associated equation for atom number variation in terms of all $x, y$, and $z$ components derived from Eq. (A9),

$$
\frac{d N(t)}{d t}=-\frac{K N^{3}}{9 \sqrt{3} \pi^{3} \hbar \mathcal{W}_{x}^{2} \mathcal{W}_{y}^{2} \mathcal{W}_{z}^{2}},
$$

as well as Eqs. (A12) and (A16), the variational equation for the condensate width along the $x$ axis is obtained by Eq. (17). Repeating the same procedure for the $y$ and $z$ components gives the variational Eqs. (18) and (19).
[1] G. Timp, R. E. Behringer, D. M. Tennant, J. E. Cunningham, M. Prentiss, and K. K. Berggren, Using light as a Lens for Submicron, Neutral-Atom Lithography, Phys. Rev. Lett. 69, 1636 (1992).

[2] J. J. McClelland, R. Scholten, E. Palm, and R. J. Celotta, Laserfocused atomic deposition, Science 262, 877 (1993).

[3] D. Meschede, Atomic nanofabrication: Perspectives for serial and parallel deposition, J. Phys.: Conf. Ser. 19, 020 (2005).
[4] V. Balykin and P. Melentiev, Nanolithography with atom optics, Nanotechnol. Russia 4, 425 (2009).

[5] V. Balykin and V. Letokhov, The possibility of deep laser focusing of an atomic beam into the å-region, Opt. Commun. 64, 151 (1987).

[6] V. I. Balykin and V. S. Letokhov, Deep focusing of an atomic beam in the Angstrom region by laser radiation, Sov. Phys. JETP 67, 150 (1988). 
[7] J. J. McClelland and M. Scheinfein, Laser focusing of atoms: A particle-optics approach, J. Opt. Soc. Am. B 8, 1974 (1991).

[8] R. Ohmukai, S. Urabe, and M. Watanabe, Atom lithography with ytterbium beam, Appl. Phys. B 77, 415 (2003).

[9] G. Myszkiewicz, J. Hohlfeld, A. Toonen, A. Van Etteger, O. Shklyarevskii, W. Meerts, T. Rasing, and E. Jurdik, Laser manipulation of iron for nanofabrication, Appl. Phys. Lett. 85, 3842 (2004).

[10] B. Smeets, P. van der Straten, T. Meijer, C. Fabrie, and K. van Leeuwen, Atom lithography without laser cooling, Appl. Phys. B 98, 697 (2010).

[11] M. K. Oberthaler and T. Pfau, One-, two-and three-dimensional nanostructures with atom lithography, J. Phys.: Condens. Matter 15, R233 (2003).

[12] E. Henn, J. Seman, G. Seco, E. Olimpio, P. Castilho, G. Roati, D. V. Magalhaes, K. M. F. Magalhaes, and V. S. Bagnato, BoseEinstein condensation in ${ }^{87} \mathrm{Rb}$ : Characterization of the Brazilian experiment, Braz. J. Phys. 38, 279 (2008).

[13] K. Ziegler and A. Shukla, Erratum: Bose-Einstein condensation in a trap: The case of a dense condensate [Phys. Rev. A 56, 1438 (1997)], Phys. Rev. A 57, 1464(E) (1998).

[14] J. J. McClelland, Atom-optical properties of a standing-wave light field, J. Opt. Soc. Am. B 12, 1761 (1995).

[15] Y. Castin and R. Dum, Bose-Einstein Condensates in Time Dependent Traps, Phys. Rev. Lett. 77, 5315 (1996).

[16] H. Bender, C. Stehle, C. Zimmermann, S. Slama, J. Fiedler, S. Scheel, S. Y. Buhmann, and V. N. Marachevsky, Probing Atom-Surface Interactions by Diffraction of Bose-Einstein Condensates, Phys. Rev. X 4, 011029 (2014).

[17] C. Stehle, H. Bender, C. Zimmermann, D. Kern, M. Fleischer, and S. Slama, Plasmonically tailored micropotentials for ultracold atoms, Nat. Photon. 5, 494 (2011).

[18] J. Mompart, V. Ahufinger, and G. Birkl, Coherent patterning of matter waves with subwavelength localization, Phys. Rev. A 79, 053638 (2009).

[19] D. Viscor, J. L. Rubio, G. Birkl, J. Mompart, and V. Ahufinger, Single-site addressing of ultracold atoms beyond the diffraction limit via position-dependent adiabatic passage, Phys. Rev. A 86, 063409 (2012).

[20] T. Judd, R. Scott, G. Sinuco, T. Montgomery, A. Martin, P. Krüger, and T. Fromhold, Zone-plate focusing of Bose-Einstein condensates for atom optics and erasable high-speed lithography of quantum electronic components, New J. Phys. 12, 063033 (2010).

[21] D. Paganin, Coherent X-ray Optics, Vol. 6 (Oxford University Press Inc., New York, 2006).

[22] P. B. Kruger, S. Mathews, K. R. Aggarwala, and N. Sanchez, Chromatic aberration and ocular focus: Fincham revisited, Vision Res. 33, 1397 (1993).

[23] J. Sasián, Introduction to Aberrations in Optical Imaging Systems (Cambridge University Press, New York, 2013).

[24] J. C. Stover, Optical Scattering: Measurement and Analysis (Society of Photo-Optical Instrumentation Engineers, Bellingham, Washington, 2012).

[25] F. Dalfovo, S. Giorgini, L. P. Pitaevskii, and S. Stringari, Theory of Bose-Einstein condensation in trapped gases, Rev. Mod. Phys. 71, 463 (1999).
[26] L. Pitaevskii and S. Stringari, Bose-Einstein Condensation (Clarendon, Oxford, 2003).

[27] C. J. Pethick and H. Smith, Bose-Einstein Condensation in Dilute Gases (Cambridge University Press, Cambridge, UK, 2002).

[28] N. N. Bogoliubov, On the theory of superfluidity, J. Phys. (USSR) 11, 23 (1947).

[29] G. D. McDonald, C. C. N. Kuhn, K. S. Hardman, S. Bennetts, P. J. Everitt, P. A. Altin, J. E. Debs, J. D. Close, and N. P. Robins, Bright Solitonic Matter-Wave Interferometer, Phys. Rev. Lett. 113, 013002 (2014).

[30] D. Murray and P. Öhberg, Matter wave focusing, J. Phys. B: At., Mol. Opt. Phys. 38, 1227 (2005).

[31] E. Braaten, H.-W. Hammer, and T. Mehen, Dilute Bose-Einstein Condensate with Large Scattering Length, Phys. Rev. Lett. 88, 040401 (2002).

[32] A. Gammal, T. Frederico, L. Tomio, and F. K. Abdullaev, Stability analysis of the $d$-dimensional nonlinear Schrödinger equation with trap and two-and three-body interactions, Phys. Lett. A 267, 305 (2000).

[33] F. K. Abdullaev, A. Gammal, L. Tomio, and T. Frederico, Stability of trapped Bose-Einstein condensates, Phys. Rev. A 63, 043604 (2001).

[34] V. M. Perez-Garcia, H. Michinel, J. Cirac, M. Lewenstein, and P. Zoller, Low Energy Excitations of a Bose-Einstein Condensate: A Time-Dependent Variational Analysis, Phys. Rev. Lett. 77, 5320 (1996).

[35] E. P. Gross, Structure of a quantized vortex in boson systems, Il Nuovo Cimento 20, 454 (1961).

[36] L. P. Pitaevskii, Vortex lines in an imperfect Bose gas, Zh. Eksp. Teor. Fiz. 40, 646 (1961) [Sov. Phys. JETP 13, 451 (1961)].

[37] E. P. Gross, Unified theory of interacting bosons, Phys. Rev. 106, 161 (1957).

[38] V. Ginzburg and L. Pitaevskii, On the theory of superfluidity, Sov. Phys. JETP 34, 858 (1958).

[39] A. J. Leggett, Bose-Einstein condensation in the alkali gases: Some fundamental concepts, Rev. Mod. Phys. 73, 307 (2001).

[40] L. Pitaevskii and S. Stringari, Bose-Einstein Condensation and Superfluidity, Vol. 164 (Cambridge University Press, New York, 2016).

[41] P. Courteille, R. S. Freeland, D. J. Heinzen, F. A. Van Abeelen, and B. J. Verhaar, Observation of a Feshbach Resonance in Cold Atom Scattering, Phys. Rev. Lett. 81, 69 (1998).

[42] N. R. Claussen, S. J. J. M. F. Kokkelmans, S. T. Thompson, E. A. Donley, E. Hodby, and C. E. Wieman, Very-highprecision bound-state spectroscopy near a $85 \mathrm{Rb}$ Feshbach resonance, Phys. Rev. A 67, 060701(R) (2003).

[43] P. J. Everitt, M. A. Sooriyabandara, M. Guasoni, P. B. Wigley, C. H. Wei, G. D. McDonald, K. S. Hardman, P. Manju, J. D. Close, C. C. N. Kuhn, S. S. Szigeti, Y. S. Kivshar, and N. P. Robins, Observation of a modulational instability in BoseEinstein condensates, Phys. Rev. A 96, 041601(R) (2017).

[44] P. A. Altin, G. R. Dennis, G. D. McDonald, D. Döring, J. E. Debs, J. D. Close, C. M. Savage, and N. P. Robins, Collapse and three-body loss in a $85 \mathrm{Rb}$ Bose-Einstein condensate, Phys. Rev. A 84, 033632 (2011).

[45] J. L. Roberts, N. R. Claussen, S. L. Cornish, and C. E. Wieman, Magnetic Field Dependence of Ultracold Inelastic Collisions Near a Feshbach Resonance, Phys. Rev. Lett. 85, 728 (2000). 
[46] R. Grimm, M. Weidemüller, and Y. B. Ovchinnikov, Optical dipole traps for neutral atoms, in Advances in Atomic, Molecular, and Optical Physics, Vol. 42 (Elsevier, San Diego, CA, 2000), pp. 95-170.

[47] J. P. Gordon and A. Ashkin, Motion of atoms in a radiation trap, Phys. Rev. A 21, 1606 (1980).

[48] D. McGloin, G. C. Spalding, H. Melville, W. Sibbett, and K. Dholakia, Applications of spatial light modulators in atom optics, Opt. Express 11, 158 (2003).

[49] L. Zhu and J. Wang, Arbitrary manipulation of spatial amplitude and phase using phase-only spatial light modulators, Sci. Rep. 4, 7441 (2014).

[50] V. M. Pérez-García, H. Michinel, J. I. Cirac, M. Lewenstein, and P. Zoller, Dynamics of Bose-Einstein condensates: Variational solutions of the Gross-Pitaevskii equations, Phys. Rev. A 56, 1424 (1997).

[51] J. Rogel-Salazar, The Gross-Pitaevskii equation and BoseEinstein condensates, Eur. J. Phys. 34, 247 (2013).

[52] W. Bao, The nonlinear Schrödinger equation and applications in Bose-Einstein condensation and plasma physics, in Dynamics in Models of Coarsening, Coagulation, Condensation and Quantization (World Scientific, Singapore, 2007), pp. 141-239.

[53] J. Söding, D. Guéry-Odelin, P. Desbiolles, F. Chevy, H. Inamori, and J. Dalibard, Three-body decay of a rubidium BoseEinstein condensate, Appl. Phys. B 69, 257 (1999).

[54] D. M. Stamper-Kurn, M. R. Andrews, A. P. Chikkatur, S. Inouye, H.-J. Miesner, J. Stenger, and W. Ketterle, Optical Confinement of a Bose-Einsteinc Condensate, Phys. Rev. Lett. 80, 2027 (1998).

[55] E. A. Burt, R. W. Ghrist, C. J. Myatt, M. J. Holland, E. A. Cornell, and C. E. Wieman, Coherence, Correlations, and Col- lisions: What One Learns About Bose-Einstein Condensates from Their Decay, Phys. Rev. Lett. 79, 337 (1997).

[56] J. Weiner, V. S. Bagnato, S. Zilio, and P. S. Julienne, Experiments and theory in cold and ultracold collisions, Rev. Mod. Phys. 71, 1 (1999).

[57] C. Kuhn, G. McDonald, K. Hardman, S. Bennetts, P. Everitt, P. Altin, J. Debs, J. Close, and N. Robins, A Bose-condensed, simultaneous dual-species Mach-Zehnder atom interferometer, New J. Phys. 16, 073035 (2014).

[58] S. L. Cornish, N. R. Claussen, J. L. Roberts, E. A. Cornell, and C. E. Wieman, Stable 85 Rb Bose-Einstein Condensates with widely Tunable Interactions, Phys. Rev. Lett. 85, 1795 (2000).

[59] M. Inguscio, S. Stringari, and C. Wieman, Bose-Einstein Condensation in Atomic Gases, Vol. 140 (IOS Press, Amsterdam, Netherlands, 1999).

[60] J. Garnier, F. K. Abdullaev, and B. B. Baizakov, Collapse of a Bose-Einstein condensate induced by fluctuations of the laser intensity, Phys. Rev. A 69, 053607 (2004).

[61] E. A. Donley, N. R. Claussen, S. L. Cornish, J. L. Roberts, E. A. Cornell, and C. E. Wieman, Dynamics of collapsing and exploding Bose-Einstein condensates, Nature (London) 412, 295 (2001).

[62] S. Balac and F. Mahé, Embedded Runge-Kutta scheme for stepsize control in the interaction picture method, Comput. Phys. Commun. 184, 1211 (2013).

[63] J. J. McClelland, Nanofabrication via atom optics, in Handbook of Nanostructured Materials and Nanotechnology (Elsevier, San Diego, CA, 2000), pp. 335-385.

[64] R. G. Scott, A. M. Martin, T. M. Fromhold, and F. W. Sheard, Anomalous Quantum Reflection of Bose-Einstein Condensates from a Silicon Surface: The Role of Dynamical Excitations, Phys. Rev. Lett. 95, 073201 (2005). 


\section{University Library}

\section{- M M I N E R VA A gateway to Melbourne's research publications}

Minerva Access is the Institutional Repository of The University of Melbourne

Author/s:

Richberg, R;Szigeti, SS;Martin, AM

Title:

Optical focusing of Bose-Einstein condensates

Date:

2021-06-14

Citation:

Richberg, R., Szigeti, S. S. \& Martin, A. M. (2021). Optical focusing of Bose-Einstein condensates. PHYSICAL REVIEW A, 103 (6), https://doi.org/10.1103/PhysRevA.103.063304.

Persistent Link:

http://hdl.handle.net/11343/283388 